\section{EMBRYRIDDLE \\ Aeronautical University}

SCHOLARLY COMMONS
International Journal of Aviation, Aeronautics, and Aerospace

\title{
Global Optimized Isothermal and Nonlinear Models of Earth's Standard Atmosphere
}

Nihad E. Daidzic, Ph.D.,

AAR Aerospace Consulting, LLC, aaraerospace@cs.com

Follow this and additional works at: https://commons.erau.edu/ijaaa

Part of the Aerospace Engineering Commons, Analysis Commons, Atmospheric Sciences Commons, Aviation Commons, Engineering Physics Commons, Numerical Analysis and Computation Commons, and the Statistical, Nonlinear, and Soft Matter Physics Commons

\section{Scholarly Commons Citation}

Daidzic,, N. E. (2015). Global Optimized Isothermal and Nonlinear Models of Earth's Standard Atmosphere. International Journal of Aviation, Aeronautics, and Aerospace, 2(3). https://doi.org/ 10.15394/ijaaa.2015.1064

This Article is brought to you for free and open access by the Journals at Scholarly Commons. It has been accepted for inclusion in International Journal of Aviation, Aeronautics, and Aerospace by an authorized administrator of Scholarly Commons. For more information, please contact commons@erau.edu. 
The existing International Standard Atmosphere (ISA) simplifies what may look as an insurmountable task of making some sense of apparently chaotic molecular motion of elementary gas particles. But even ISA is often clumsy, cumbersome, and complicated for quick estimates and especially so for higher altitudes. Since ISA consists of several discrete temperature layers (ICAO, 1993; ISO, 1975; NOAA, 1976) still complex computations are required despite recent efforts to design a general computational algorithm that facilitates and simplifies computations for arbitrary altitudes below 86 orthometric kilometers (Daidzic, 2015). The reasons for introduction of ISA were already discussed in a recent article by Daidzic (2015) and will thus not be repeated here. Existing international standards of Earth's atmosphere cover the range of altitudes from the Mean Sea Level (MSL) up to $80 \mathrm{~km}$ (ICAO, 1993) or up to 1,000 km (ISO, 1975; NOAA, 1976). The basic temperature layers of the ISA homosphere with the associated Temperature Lapse Rates (TLR) are presented in Table 1.

Table 1

Atmospheric Temperature Layers of ISA Homosphere

\begin{tabular}{lcc}
\hline $\begin{array}{c}\text { Atmospheric Layer } \\
\text { (Homosphere) }\end{array}$ & $\begin{array}{c}\text { Altitude Range } \\
\text { (Geopotential) }[\mathrm{km}]\end{array}$ & $\begin{array}{c}\text { TLR } \\
d T / d H[\mathrm{~K} / \mathrm{m}]\end{array}$ \\
\hline Troposphere & $0-11$ & -0.0065 \\
Tropopause (SS I) & $11-20$ & 0 \\
Stratosphere II & $20-32$ & +0.001 \\
Stratosphere III & $32-47$ & +0.0028 \\
Stratopause (MS I) & $47-51$ & 0 \\
Mesosphere II & $51-71$ & -0.0028 \\
Mesosphere III & $71-84.852$ & -0.0020 \\
\hline
\end{tabular}

Above the homosphere, in which the homogeneous perfect-gas air mixture presents a reasonable approximation due to intense mixing and local convective overturning, the in-homogeneous heterosphere is located. Heterosphere consists of thermosphere (up to $500 \mathrm{~km}$ ) and exosphere (from 500 to $1,000 \mathrm{~km}$ ) and must be treated as a real gas. Ozone layer forms in stratosphere (20-30 km) photochemically (Iribarne \& Cho, 1980). The air temperature in thermosphere, which now experiences increasing dissociation and ionization (Pai, 1981), undergoes wild $500^{\circ}-800^{\circ} \mathrm{C}$ diurnal oscillations due to variable absorption of sun's radiation (Iribarne \& Cho, 1980). Ionization generates several ionospheric layers that migrate diurnally and much of it depends on the solar activity. Diffusion, photoelectric, and photochemical processes become very important. Temperatures 
of the rarefied thermosphere air can reach 1,500 to 2,000 Kelvin (K) during peak radiation absorption cycles.

The exosphere or the "spray region" is regarded as the final layer of the Earth's atmosphere and the transition from the terrestrial atmosphere to interplanetary gas. Significantly fewer collisions between the ions, atoms, and molecules take place in exosphere. The base of the exosphere is defined as the condition in which the average MFP is equivalent to the local scale height of the atmosphere (i.e., $500 \mathrm{~km}$ ). Free molecular flow exists and the gravitational and electromagnetic forces are dominating particle dynamics with the very low collision frequencies. Some of the elementary particles will join the Earth's orbit, some will exhibit ballistic trajectories ultimately returning to Earth's atmosphere, and some may entirely escape Earth's gravitational pull.

However, although homospheric ISA simplifies the real atmosphere considerably and provides a standard for aircraft performance testing, it is still quite complicated, bulky, and computation intensive. Many ISA temperature layers with discontinuous TLRs complicates the issue. It can also be stated that ISA represents neutrally stable atmosphere at standard (negative) TLR of $1.98^{\circ} \mathrm{C} / 1000 \mathrm{ft}$, which is located between the Dry Adiabatic Lapse Rate (DALR) of $3^{0} \mathrm{C} / 1000 \mathrm{ft}$ and the Saturated Adiabatic Lapse Rate (SALR), which according to Dutton (2002) typically can be taken as $1.5^{\circ} \mathrm{C} / 1000 \mathrm{ft}$ in mid-latitudes.

The basic motivation behind this article is to introduce simpler atmospheric models, which conserve the total atmospheric mass and weight. In that respect, an Isothermal Atmospheric Model (ISOAM) and the Nonlinear Parabolic Atmospheric Model (NLPAM), which best approximate the ISA's TLR were developed making estimation of vertical pressure and density distribution much simpler. This is especially true for the upper atmospheric layers where twelve or fourteen pressure and density functions are replaced by just one expression for temperature and one for pressure or density. The ideal-gas law then connects the three thermodynamic variables in a unique fashion.

Two important heights/altitudes exist in Earth's atmosphere relevant to the aviation and aerospace industries. One is the Armstrong (physiological) limit (named after Harry George Armstrong) which is located at about $19.2 \mathrm{~km} \mathrm{(63,000}$ $\mathrm{ft}$ ) and designates the height at which the total atmospheric pressure drops to less than $63 \mathrm{hPa}(0.91 \mathrm{psi})$ at which the unprotected (unpressurized) human bodily fluids would start evaporating/boiling considering the normal average human body temperature of about $37^{\circ} \mathrm{C}$ (Daidzic \& Simones, 2010). About $70 \%$ of human body is water and many different bodily fluids have essential life- 
preservation functions. In reality, the blood is circulating under somewhat higher (gage) pressure and it would take some time and even lower atmospheric pressures to start ebulism and anoxia (Daidzic \& Simones, 2010). This is the main reason pressurized suits must be worn above 50,000 feet (aeromedical "onset of space") unless the aircraft is built to stricter space standards. Armstrong's limit sets restrictions to commercial passenger transportation.

Another important limit is the von Kármán (aerodynamic) limit (named after Theodore von Kármán) which is located at around $100 \mathrm{~km}$ (about 330,000 feet) and often, designated as the "beginning of space". The air density at these altitudes is so low that an aircraft would have to move at speeds equal to or higher than about $7.8 \mathrm{~km} / \mathrm{s}$ to generate enough lift to sustain flight by Angle-of-Attack (AOA). That speed would correspond to circular orbital speeds of Low Earth Orbits (LEO). Von Kármán limit is thus the aerodynamic limit of atmospheric flight by AOA and the concept could be extended to other planetary atmosphere.

The main application areas of the two new ISA approximations is in airplane testing and design, calculation of drag for supersonic, hypersonic, and trans-atmospheric vehicles and easier computations of inverse problems. Approximate analytical solutions could greatly benefit from simpler atmospheric models. While ISA is standard, the computations must be done marching in space from SL up to and including the particular layer. The main motivation behind ISOAM is in simpler computations of drag from SL all the way up to von Kármán limit. In fact, the first rough estimates of drag for the slip and free-molecular atmospheric regions could be obtained by extending ISOAM up to $150-300 \mathrm{~km}$. For trans-atmospheric flights, ballistic missiles, and space launches and reentries is the simple model of atmosphere important in the approximate estimation of optimal trajectories. ISOAM can be extended to other planetary atmospheres (e.g., Venus, Jupiter) especially in terms of entry/re-entry problems. On the other hand, the NLPAM can be used as a reliable ISA substitute for altitudes up to $47 \mathrm{~km}$.

\section{Literature Review}

Historically, many attempts were made to construct simple atmospheric models. Often the first choice was in designing isothermal atmosphere. Less frequent were models of the linear atmosphere in which the temperature is linearly changing with height. While the troposphere definitely experiences global negative TLR, despite some localized temperature inversions (positive TLRs), the rest of the ISA has several isothermal layers and the globally positive TLR regions (upper stratosphere) for which a negative TLR would be absolutely 
inappropriate. In order to model the TLR throughout the ISA troposphere and stratosphere, a single parabolic NLPAM TLR is introduced.

The issue of an exponential atmosphere finds its origins in the kinetic theory of gases in gravitational field. One of the first considerations elucidating that was made by Kennard (1938). The problem is that gravitational forces are not the only ones exerting influence on gas particles and thus an isothermal atmosphere is an idealization. One of the first applications of an isothermal atmospheric standard was given by Chapman (1958) in regards to planetary atmospheric (re)entry of space vehicles. The initial problem of that time was of course in resolving the issue of reentry into Earth atmosphere of ballistic missiles and the reentry of manned space capsules and satellites, which was just in its early phase. Steep reentry angles and enormous heat loads were (and still are) a big concern. Chapman used exponential atmospheric model to then solve differential equations for entry into atmospheres of Earth, Mars, Venus, and Jupiter. Ashley (1992) uses an isothermal atmospheric model which approximates ISA when considering space launch and reentry vehicles (ballistic missiles and satellites). His model is based on the Chapman model (Chapman, 1958). Tewari (2007) also used an isothermal model of atmosphere based on the least-square optimization although details of it were not shown. According to Tewari, the approximation is reasonably good for the homosphere, but not for upper atmospheric layers. However, Tewari's resulting Sea Level (SL) density is extreme and the model is not recommended below $5 \mathrm{~km}$. Similar isothermal models are used in many atmospheric physics books (e.g., Dutton, 2002; Houghton, 2000; Iribarne \& Cho, 1980; Wallace \& Hobbs, 2006).

However, the problem with previous isothermal atmospheres is that the base density $\rho_{0}$ often does not correspond to ISA SL density. As a matter of fact in order to fit the data, the SL density in exponential (isothermal) models is sometimes almost 50\% higher than ISA's SL density as in Tewari (2007). The problem with all these earlier works is they used exponential functions with two degrees of freedom (independent coefficients to be optimized) in their models of the exponential form $\rho=\rho_{0} \exp \left(-\kappa^{*} \cdot H\right)$.

The base density actually is a function of the isothermal temperature constrained by the ideal-gas law once the standard SL pressure is chosen (e.g., ISA SL). Accordingly, such a constrained problem cannot be solved using the methods of linear least-squares as the base-density is dependent on the temperature in the scale factor $\kappa^{*}=g_{0} / R T_{0}$. In other words, the SL temperature $T_{0}$ affects both coefficients $\left(\rho_{0}\right.$ and $\left.\kappa^{*}\right)$ which then become dependent. One then 
has to deal with the problem of nonlinear regression (best-fit) which is far more complicated (Chapra \& Canale, 2006; Press et al., 1992). Therefore, the ISOAM model introduced here is starkly different from the previous global exponential models of atmosphere by the fact that SL constraint will be imposed on the air properties (pressure, temperature, and density). No reference to a nonlinear TLR model (such as NLPAM) was found and to the best of my knowledge this is the first time such considerations are given.

Mass of the atmosphere and surface pressures of dry and humid air as a function of season, hemispheric (North and South) latitudes, and averaged for the entire globe were given in several articles by Trenberth at coworkers (Trenberth, 1981, Trenberth \& Guillemot, 1994; Trenberth \& Smith, 2005). The average surface elevation (topography) varied in various estimates but most of the results are between 231 and $239 \mathrm{~m}$ (758 and $784 \mathrm{ft})$. The MSL elevation of about $70 \%$ of Earth's surface is zero. Trenberth (1981) estimated the average SL pressure to be $1011.00 \mathrm{hPa}$ based on the averages over the Northern $(\mathrm{NH})$ and the Southern $(\mathrm{SH})$ hemisphere and over the months of January and July. Trenberth and Guillemot (1994) provided an update on surface pressure data and atmospheric mass and found that the average atmospheric mass is $5.1441 \times 10^{18} \mathrm{~kg}$. The global mean water vapor pressure was $2.58 \mathrm{hPa}$ with an annual cycle range of $0.36 \mathrm{hPa}$ which enables the calculation of the moisture content in atmosphere and monitoring its changes spatially and temporally. The total dry-air atmospheric mass was $5.132 \times 10^{18} \mathrm{~kg}$ (Trenberth \& Guillemot, 1994). The average total surface pressure according to latest update by Trenberth and Smith (2005) for measurements conducted in a period 1979-2001 is $985.5 \mathrm{hPa}$ for humid- and $983.05 \mathrm{hPa}$ for dryair (such as in ISA model). Considering the average height of topography (MSL), the difference between SL and surface pressures is less than $30 \mathrm{hPa}$, but this varies from the equatorial to the polar region and over seasonal cycles. At lower altitudes only, it is assumed that each $10 \mathrm{~m}$ of vertical air-column is equivalent to about $1.2 \mathrm{hPa}$. The ISA standard SL pressure is $1013.25 \mathrm{hPa}$ and corresponds roughly to an average measured SL pressure at $20^{\circ}$ latitude in $\mathrm{NH}$. The average atmospheric mass of dry air is according to Trenberth and Smith (2005) equal to $(5.1352 \pm 0.0003) \times 10^{18} \mathrm{~kg}$. The mass of moisture in atmosphere is about two-tothree orders-of-magnitude lower, i.e., for each $\mathrm{kg}$ of atmospheric water there is $400 \mathrm{~kg}$ of dry air.

\section{Mathematical Model of Atmosphere}

Knowledge of atmospheric parameters, such as, temperature, density and pressure for arbitrary orthometric altitudes is crucially important in aviation and aeronautical industry. Pressure altimeter calibration, flight testing and 
performance data scaling and processing are some of the applications where the mutually-agreed standard atmospheric model is needed.

\section{Discrete Nature of Atmosphere}

The Earth's atmosphere consists of huge number of elementary particles (molecules, atoms, and ions) in thermal motion. Tracking all these particles in time and space is practically and theoretically an impossible task. Fortunately it is not needed. One of the most important physicists of the $19^{\text {th }}$ century, Ludwig Boltzmann was also one of the founding fathers of the gas theory, pre-quantum atomic theories, and the statistical physics as evident from his original masterpiece work in 1896 and 1898 (Boltzmann, 1964). The classical MaxwellBoltzmann (MB) statistics being a limiting case of quantum Bose-Einstein (BE) and Fermi-Dirac (FD) statistics (Hansen, 1976; Hill, 1987; Holman, 1980; Reif, 1965; Sears, 1964; Tribus, 1961; Wannier, 1987) predicts the distribution of molecular velocities in an ideal dilute gas quite well, which has also been confirmed by numerous experiments. The kinetic theory of gases (Holman, 1980; Kennard, 1938; Reif, 1965; Saad, 1966; Sears, 1964) describes transport processes of gas particles in thermal motion remarkably well. A Mean Free Path (MFP), collision frequency, momentum exchange, etc., define various mesoscopic transport properties such as dynamic viscosity and the coefficient of heat conduction. For example, a single "anonymous" diatomic nitrogen molecule $\left(\mathrm{N}_{2}\right)$ at SL pressure and temperature of $300 \mathrm{~K}\left(27^{0} \mathrm{C}\right)$ will have MFP of $\lambda=58$ nanometer $(\mathrm{nm})$ and the collision frequency of almost nine billion impacts per second $(9 \mathrm{GHz})$ with other anonymous nitrogen molecules. That implies about one collision every 0.1 nanosecond (ns). On average every $\mathrm{N}_{2}$ molecule will "miss" 9 other anonymous molecules before colliding with the $10^{\text {th }}$ under given conditions. Almost $10^{20}$ nitrogen molecules will be found in one cubic centimeter $\left(\mathrm{cm}^{3}\right)$ of air at SL pressure and $300 \mathrm{~K}$. A Root-Mean-Square (RMS) speed of a nitrogen molecule is about 517 meters per second $(\mathrm{m} / \mathrm{s})$ or about 1005 knots at SL pressure and temperature of $300 \mathrm{~K}$. A single $\mathrm{N}_{2}$ molecule has a diameter of about $0.4 \mathrm{~nm}$. Thus a MFP, as expected for a continuum, is on average 100 times larger than the representative size of a molecule. The molecular spacing is about 10 times the molecule size. However, about 45-in-a-million nitrogen molecules will have MFPs ten times longer (about $600 \mathrm{~nm}$ ). Nevertheless, this length scale is still much smaller than the characteristic length scales in atmospheric flows.

The non-dimensional Knudsen number $\left(K n=\lambda / l^{*}=M / \sqrt{\operatorname{Re}}\right)$, which is the ratio of the molecular MFP and the macroscopic (integral) flow scale, is extremely small in this case justifying the continuum assumption. The kinetictheory of gases shows that the MFP is inversely proportional to the gas pressure. 
As the pressure decreases by a factor of $10^{5}$ so does the MFP increase by a factor of $10^{5}$. For typical characteristic length scales in atmospheric flows one could, for example, deduce the size of the Kolmogorov eddies (micro-scale) to be on the order of one millimeter $(\mathrm{mm})$. Typically, atmospheric Kolmogorov eddies responsible for atmospheric flows viscous dissipation have sizes that are on the order of 100 micrometer ( $\mu \mathrm{m}$ is $10^{-6} \mathrm{~m}$ ) to $10 \mathrm{~mm}$ and represent the smallest aerodynamic scales in the respective gaseous fluid continuum. Thus, $K n<<0.01$, justifying the continuum concept. The hydrodynamic continuum limit may be even two-orders of magnitude smaller than the aerodynamic due to much higher densities and shorter intermolecular spacing in liquids. Kolmogorov eddies are responsible for the final viscous dissipation of turbulent motion and conversion of flow energy into heat (Daidzic, 1992a, 1992b; Tennekes \& Lumley, 1980). A ratio of MFP and the Kolmogorov-eddy scale is $K n=\lambda / \eta=M / \sqrt[4]{\operatorname{Re}}$ (Tennekes $\&$ Lumley, 1980). Dissipation rate of turbulent energy is an essential part of the turbulent energy transport and its measurements are crucial in understanding turbulent scales and dynamics (Azad \& Kassab, 1980).

The universal gas constant $\mathfrak{R} \approx 8,314 \mathrm{~J} /(\mathrm{kmol} \cdot \mathrm{K})$, the Avogadro number $N_{A} \approx 6.022 \times 10^{26} \mathrm{kmol}^{-1}$ and the Boltzman constant $k_{B} \approx 1.38 \times 10^{-23} \mathrm{~J} / \mathrm{K}$ were used in basic molecular dynamics estimates here. In order to arrive at these estimates, calculations using the MB statistics and the kinetic theory of gasses were implemented (Reif, 1965; Sears, 1964). Details are not shown as that would distract us from the main objective. This short example is solely intended to illustrate the enormous complexity of the micro-world and the need to come up with theories that can be reasonably handled and deliver meaningful results.

\section{Continuum Model of Atmosphere}

The continuum (low Knudsen-number) fluid mechanics (Chadwick, 1999; Landau \& Lifshitz, 1987) entirely neglects the individuality of particles in thermal motion and introduces statistically-averaged intensive thermodynamic variables such as pressure, density, and temperature of air parcels containing huge number of molecules. Fortunately, these three essential thermodynamic properties of dilute gas mixtures are connected through a remarkably simple relationship - the ideal gas law (Holman, 1980; Saad, 1966; Sears, 1964; Tribus, 1961). The change of atmospheric pressure is thus based on the fundamental equation of aerostatics (Daidzic, 2015; Dutton, 2002; Iribarne \& Cho, 1980; Pai, 1981):

$$
d p(z)=-\rho(z) \cdot g(z) \cdot d z=-\left[\frac{p \cdot M(z)}{\mathfrak{R} \cdot T(z)}\right] \cdot g(z) \cdot d z=-\left[\frac{p}{R(z) \cdot T(z)}\right] \cdot g(z) \cdot d z
$$


This equation is strictly valid for vertical pressure distribution over flat surfaces. In curved geometries the hydrostatic fluid pressure does not equal the weight per unit area of the fluid above it (Ambaum, 2008). According to the same author a geometric reduction in surface pressure as large as $5 \mathrm{hPa}$ (mbar) exists for Earth. An ideal-gas law can be written at any height as:

$\ln p=\ln \rho+\ln R+\ln T \Rightarrow \frac{d p}{p}=\frac{d \rho}{\rho}+\frac{d T}{T} \quad R=$ const.

Hence, for isothermal atmosphere, the relative pressure and density changes are equivalent. The air gas constant $R=\mathfrak{R} / M$ can only be a function of height due to changes in molecular weight (above 80-86 km). Furthermore, it is assumed that aerostatic and thermodynamic pressures are equal in the absence of vertical acceleration and that atmospheric pressure is only a function of height (Dutton, 2002). As has been reported earlier (NOAA, 1976), the average molecular mass of dry air mixture stays essentially constant up to 86 orthometric $\mathrm{km}$. That is due to intense mixing and localized convective overturning that prevents heavier gas components to settle in lower atmosphere and lighter components in upper atmosphere (Iribarne \& Cho, 1980). The gravitational acceleration for a spherical uniform-density Earth as a function of orthometric height is:

$$
g(z)=g_{o}\left(\frac{R_{0}}{R_{0}+z}\right)^{2} \quad g_{o}=9.80665 \mathrm{~m} / \mathrm{s}^{2} \quad R_{0}=6,371 \mathrm{~km}
$$

As will be seen in a subsequent article on Earth's shape and gravity, the SL-average gravitational attraction $g_{o}$ is actually a function of latitude $g=g(\phi)$ (neglecting tesseral gravitational anomalies). In terms of the geopotential height, the Equation 1 now becomes:

$$
d p=-\left[\frac{p \cdot M(H)}{\mathfrak{R} \cdot T(H)}\right] \cdot g_{0} \cdot d H=-\left[\frac{p}{R(H) \cdot T(H)}\right] \cdot g_{0} \cdot d H
$$

Where the thermal and aerostatic equilibrium at lower altitudes imply the following relationship between the molecular-scale and thermodynamic (kinetic) temperature (NOAA, 1976; Tewari, 2007): 
$T(H)=T_{t h}(H) \frac{M_{0}}{M(H)}$

The relationships between the constant-g (geopotential) height and the orthometric (geometric or MSL) height are (Daidzic, 2015):

$$
H=\left(\frac{R_{0}}{R_{0}+z}\right) \cdot z \quad z=\left(\frac{R_{0}}{R_{0}-H}\right) \cdot H \quad|H| \leq|z|
$$

For the constant-TLR homosphere (troposphere, stratosphere, mesosphere, etc.), and the constant gravitational acceleration, the change in atmospheric pressure with geopotential height follows the differential law:

$\frac{d p}{p}=-\left[\frac{M(H) \cdot g_{0}}{\mathfrak{R} \cdot T(H)}\right] \cdot d H=-\left[\frac{g_{0}}{R(H) \cdot T(H)}\right] \cdot d H$

We may also write:

$$
\int_{p_{0}}^{p} \frac{d p}{p}=\int_{\delta_{0}}^{\delta} \frac{d \delta}{\delta}=\ln \left(\frac{\delta}{\delta_{0}}\right)=\int_{0}^{H} f(H ; R, T) d H \Rightarrow \delta(H)=\delta_{0} \cdot \exp \left[\int_{0}^{H} f(H ; R, T) d H\right]
$$

By definition pressure ratios are: $\delta=\frac{p}{p_{S L}^{S T D}} \quad \delta_{0}=\frac{p_{0}}{p_{S L}^{S T D}}$.

This accounts for the fact that an arbitrary atmospheric standard may have SL pressure, density, and temperature different from ISA's definitions. However, from the three thermodynamic properties, one is always dependent (constrained) by the other two by the ideal-gas law. The vertical pressure distribution is obtained by integration of Equation 6 from SL to an arbitrary geopotential height:

$$
\delta(H)=\delta_{0} \cdot \exp \left\{-g_{0} \cdot \int_{0}^{H} \frac{M(H) \cdot d H}{\Re \cdot T(H)}\right\}=\delta_{0} \cdot \exp \left\{-g_{0} \cdot \int_{0}^{H} \frac{d H}{R(H) \cdot T(H)}\right\}
$$

In order to solve the integral in Equation 7, the knowledge of molecularscale temperature profiles (NOAA, 1976; Tewari, 2007) with height is required. With good approximation it can be assumed that thermodynamic and molecularscale temperatures are equivalent in the homosphere. The variation of molecular 
weight or air gas "constant" with geopotential height is also required. Generally, Equation 7 would be the most appropriate when modelling pressure changes in the thermosphere. Additionally, due to its strong dependence on solar irradiation, no steady-state temperature or pressure distribution is appropriate.

However, according to Iribarne \& Cho (1980) and NOAA (1976), the Earth atmosphere is very well mixed below about $80-100 \mathrm{~km}$ and according to ISA standard it can be assumed that the average molecular weight of dry air is constant below about $86 \mathrm{~km}\left(M=M_{0}=28.9644 \mathrm{~kg} / \mathrm{kmol}\right)$ and the molecularscale and thermodynamic temperatures are identical. Utilizing Equations 4 and 7, this results in significantly simpler formulation where air gas constant is essentially independent of altitude (up to $86 \mathrm{~km}$ ):

$$
\delta(H)=\delta_{0} \cdot \exp \left\{-\left(\frac{g_{0}}{R}\right) \cdot \int_{0}^{H} \frac{d H}{T(H)}\right\}
$$

This is quite a general formulation of pressure distribution in homosphere and is valid for an arbitrary vertical temperature profile $T(H)$. It has been shown in great detail how to integrate Equation 8 for multiple ISA homospheric layers (Daidzic, 2015). Accordingly, the two basic models of the molecular-scale temperature change exist in ISA: isothermal (with the constant temperature or zero TLR) and linear (constant positive or negative TLR). In the case of simple ISOAM, where $T(H)=T_{0}$, one obtains (where, $\delta_{0}=\sigma_{0} \theta_{0}$ ):

$$
\delta(H)=\delta_{0} \exp \left\{-\frac{g_{0}}{R T_{0}} \int_{0}^{H} d H\right\}=\delta_{0} \exp \left\{-\left(\frac{g_{0}}{R T_{0}}\right) \cdot H\right\}=\delta_{0} \exp \left(-\kappa^{*} H\right)
$$

Additionally, if such atmosphere assumes SL pressure to equal ISA's SL pressure, then $\delta_{0}=1$. The subscript " 0 " refers to lowest (base) layer (in ISA it is the troposphere). If, on the other hand, the TLR is constant or the vertical temperature profile has constant slope it will follow the linear law:

$$
T(H)=T_{0}+\alpha_{0} H \quad \theta(H)=\theta_{0}\left(1+\frac{\alpha_{0}}{T_{0}} H\right) \quad \theta=\frac{T(H)}{T_{S L}^{I S A}} \quad \theta_{0}=\frac{T_{0}}{T_{S L}^{I S A}}
$$

Here, we allowed for the base surface temperature $T_{0}$ to be different than the ISA's standard surface temperature, which is captured by the temperature 
correction factor $\theta_{0}$ ( $\theta$ is temperature ratio). TLR can be positive, negative or zero, i.e., $\alpha_{0}=(d T / d H)_{0} \leq \geq 0$. The pressure distribution versus geopotential height for linear atmosphere (Equation 10) substituted into Equation 8 yields:

$$
\delta(H)=\delta_{0} \exp \left\{-\left(\frac{g_{0}}{R}\right)_{0}^{H} \frac{d H}{T_{0}+\alpha_{0} H}\right\}=\delta_{0} \exp \left\{-\left(\frac{g_{0}}{\alpha_{0} R}\right) \cdot \ln \left(1+\frac{\alpha_{0}}{T_{0}} H\right)\right\}
$$

Which reduces to the familiar constant-TLR pressure law or Linear Atmospheric Model (LAM):

$$
\delta_{0-H}(H)=\delta_{0} \cdot\left(1+\frac{\alpha_{0}}{T_{0}} H\right)^{-\left(\frac{g_{0}}{\alpha_{0} R}\right)}=\delta_{0} \cdot\left(\frac{\theta}{\theta_{0}}\right)^{-\lambda_{0}} \quad \lambda_{0} \equiv\left(\frac{g_{0}}{\alpha_{0} R}\right)
$$

More complex TLR laws can be constructed. If the vertical temperature profile of atmosphere includes low-altitude inversion with temperatures initially increasing before starting global decrease, a simple parabolic law produces:

$$
T(H)=a_{0}+a_{1} H+a_{2} H^{2} \quad a_{0}>0 \quad a_{1}>0 \quad a_{2}<0
$$

The pressure distribution as a function of geopotential height with the discriminant, $D=a_{1}^{2}-4 a_{0} a_{2}$, becomes (Dwight, 1961; Spiegel \& Liu, 1999):

$$
\begin{aligned}
\delta(H)= & \delta_{0} \cdot \exp \left\{-\left(\frac{g_{0}}{R}\right) \cdot \int_{0}^{H} \frac{d H}{a_{0}+a_{1} H+a_{2} H^{2}}\right\}= \\
& =\delta_{0} \cdot \exp \left\{\begin{array}{ll}
-\left(\frac{g_{0}}{R}\right) \cdot\left[\frac{2}{\sqrt{-D}} \tan ^{-1}\left(\frac{a_{1}+2 a_{2} H}{\sqrt{-D}}\right)\right]_{0}^{H} & D<0 \\
-\left(\frac{g_{0}}{R}\right) \cdot\left[\frac{1}{\sqrt{D}} \ln \left(\frac{a_{1}+2 a_{2} H-\sqrt{D}}{a_{1}+2 a_{2} H+\sqrt{D}}\right)\right]_{0}^{H} & D>0
\end{array}\right\}
\end{aligned}
$$

For the special case when $D=0$, one obtains (Dwight, 1961): 


$$
\begin{aligned}
\delta(H) & =\delta_{0} \cdot \exp \left\{-\left(\frac{g_{0}}{R}\right) \cdot \int_{0}^{H} \frac{d H}{a_{0}+a_{1} H+a_{2} H^{2}}\right\}= \\
& =\delta_{0} \cdot \exp \left\{-\left(\frac{g_{0}}{R}\right) \cdot\left(\frac{-2}{a_{1}+2 a_{2} H}\right)_{0}^{H}\right\}
\end{aligned}
$$

Even more complicated temperature profiles can be devised to approximate ISA with one functional relationship, but these efforts reach the point of diminished returns as they could become more complicated than ISA itself. In general, the integral defined in Equation 8 can be computed numerically for arbitrary well-behaved temperature profiles. If the vertical temperature profile comes from vertical sounding (measurements), then numerical integration delivers vertical pressure and density distributions of the still atmospheric air. The non-dimensional air mass density for a given layer, utilizing the non-dimensional ideal-gas law (constitutive relationship) is:

$\sigma_{n}(H)=\frac{\delta_{n}(H)}{\theta_{n}(H)}$

In the actual analysis of the ISA, ISOAM, LAM, and NLPAM it is assumed that the Earth is a perfect sphere of uniform mass-density with the gravitational equipotential surfaces being concentric spheres and the gravitational acceleration vectors being equivalent to the radius vectors emanating from the geocenter and barycenter. Above $86 \mathrm{~km} \mathrm{MSL}$, the physical-chemical processes in the Earth's atmosphere (real gases) become much more complex and relatively simple modeling using the ideal-gas equation is no longer valid (NOAA, 1976).

\section{Methods and Materials}

Despite great simplifications introduced by standard-atmosphere model compared to the real atmosphere, ISA is still very complex and often impractical to use. Different functional relationships for vertical pressure and density distribution must be used in conjunction with the particular layer temperature distribution. One often needs just a simple expression to determine pressure and densities for arbitrary heights. Can a simpler model of ISA be constructed? Could a global atmospheric model be based on an ISOAM or LAM at least up to $86 \mathrm{~km}$ ?

Indeed, two models of ISA atmospheric model will be now introduced with the main goal to simplify and approximate the ISA. As will be seen later, both models provide fairly accurate predictions of vertical pressure and density 
distribution in troposphere and stratosphere (up to $47 \mathrm{~km}$ ). The ISOAM is particularly simple and convenient. The ISOAM model can be extended to any arbitrary altitude, while the NLPAM, as derived here, is only valid up to $47 \mathrm{~km}$. However, the nonlinear NLPAM delivers more realistic vertical temperature profile and more accurate pressure and density distributions than ISOAM.

\section{Global Isothermal Atmospheric Model}

Considering that vertical temperature distribution meanders throughout ISA perhaps a constant-temperature atmosphere up to $86 \mathrm{~km}$ could be found that approximates ISA well. But there are infinite number of possible isotherms. The choice of the uniform temperature from SL up to $86 \mathrm{~km}$ is based here on numerically minimizing the $\mathrm{L}^{2}$ norm. Theoretically, the $\mathrm{L}^{2}$ norm is the square-root of the (Lebesgue) integral of the square of the absolute difference between the ISA and ISOAM mass-densities at every vertical point. In this way the mass of the atmosphere is best conserved. The ISOAM SL density is then a function of ISOAM SL pressure and the global isothermal temperature constrained by the ideal-gas law. ISOAM model theoretically extends atmosphere to infinity, but practically the exponential dependence will lead to a relatively rapid convergence to zero. The whole optimization procedure was done numerically and in an iterative manner until the best-fitting isothermal temperature was obtained. The new ISOAM of the homosphere drastically simplifies the multi-layered ISA model. Masses, weights, and scale heights can be easily determined from ISOAM.

However, it must be understood that ISOAM is just a very practical and simple substitute to considerably more complicated ISA model. In terms of the geopotential altitude the ISOAM problem is formulated as (Equations 2 and 9):

$$
\sigma_{\text {ISOAM }}(H)=\sigma_{0} \exp \left(-\kappa^{*} H\right) \quad \delta_{\text {ISOAM }}(H)=\delta_{0} \exp \left(-\kappa^{*} H\right) \quad 0 \leq H<\infty
$$

with: $\delta_{0}=\frac{p_{0}}{101,325} \quad \theta_{0}=\frac{T_{0}}{288.15} \quad \sigma_{0}=\frac{\rho_{0}}{1.225}=\frac{\delta_{0}}{\theta_{0}}$

The all-important ISOAM scale-height is defined as (Dutton, 2002):

$H^{*} \equiv \frac{1}{\kappa^{*}}=\frac{R \cdot T_{0}}{g_{0}} \quad T_{0}=T_{I S O A M}=$ const

The unknown optimum ISOAM constant temperature $T_{0}$ is found from the minimization of the following $\mathrm{L}^{2}$-norm: 


$$
\begin{aligned}
e_{\rho}(T)= & \min \left(\int_{0}^{\infty}\left|\rho_{I S A}(h)-\rho_{\text {ISOAM }}(h)\right|^{2} d h\right)^{1 / 2}= \\
& =\min \left\{\int_{0}^{\infty}\left|\sigma_{I S A}(h)-\sigma_{0} \exp \left(-h / H^{*}\right)\right|^{2} d h\right\}^{1 / 2}
\end{aligned}
$$

The following conditions on integrals (Lebesgue or Riemann) must be satisfied:

$$
\int_{0}^{\infty}\left|\sigma_{I S A}(h)\right| d h<\infty \quad \int_{0}^{\infty}\left|\sigma_{0} \exp \left(-h / H^{*}\right)\right| d h<\infty
$$

These condition are indeed satisfied as the atmospheric mass is finite and simpler Riemann integral does exist. As will be seen later the representative isothermal temperature is rounded to integer value of $275 \mathrm{~K}$ for easier usage. The exact value is closer to $274.605 \mathrm{~K}$, but that is impractical for regular use. The minimum is quite shallow around $274.5-275.5 \mathrm{~K}$ and the error in rounding it to an integer temperature value is minimal.

Integrals given in Equation 18 are cumbersome as we go from one atmospheric layer to another. It is thus more convenient to find a solution based on discrete values and in this case the performance measure being a sum of density difference squares must be minimized:

$$
S=\sum_{i=1}^{N}\left[\sigma_{I S A}\left(H_{i}\right)-\sigma_{I S O A M}\left(H_{i}\right)\right]^{2}
$$

This would seem to be the case of simple linear regression where the exponential form (Equation 17) is first linearized through taking the logarithm, $\ln \sigma=\ln \sigma_{0}-\kappa^{*} \cdot H$, but in fact the optimization is constrained by the SL density which is the function of SL base pressure and isothermal temperature (ISOAM). Thus this becomes a problem of nonlinear regression (nonlinear least-squares) and the Gauss-Newton iterative method is often used to solve it (Chapra \& Canale, 2006). Details of the methodology are given in Appendix A.

The ISOAM has important application in probing planetary atmospheres during space vehicle atmospheric entry. If gravitational acceleration is neglected, the initial deceleration during atmospheric entry becomes (Tewari, 2007): 
$-\frac{d v}{d t}=\frac{1}{2} \cdot \rho_{0} \exp \left(-h / H^{*}\right) \cdot v_{e}^{2} \frac{C_{D} A}{m}$

A probe entering the atmosphere uses radar to measure height and closure rate. Since the ballistic coefficient $\left(B C=m / C_{D} A\right)$ and the entry speeds $v_{e}$ are known, the approximate base density $\rho_{0}$ and isothermal atmosphere scale height $H^{*}$ can be estimated without performing extensive atmospheric measurements.

\section{Nonlinear Atmospheric Model}

A nonlinear parabolic model of ISA troposphere and stratosphere or NLPAM will be now constructed. International standard of troposphere and stratosphere has 4 layers of which one is isothermal (tropopause), one has negative TLR, and two upper stratospheric layers have two different positive TLRs (NOAA, 1976). A nonlinear TLR parabolic law approximating ISA troposphere and stratosphere results in:

$T(H)=a_{0}+a_{1} H+a_{2} H^{2} \quad a_{0}>0 \quad a_{1}<0 \quad a_{2}>0$

In dimensionless form it yields:

$$
\theta(H)=\theta_{0}\left(1+a_{1}^{*} H+a_{2}^{*} H^{2}\right) \quad \theta_{0}=\frac{a_{0}}{T_{0}} \leq \geq 1 \quad a_{1}^{*}=\frac{a_{1}}{T_{0}} \quad a_{2}^{*}=\frac{a_{2}}{T_{0}}
$$

In order to find unknown coefficients: $a_{0}$ (SL temperature), $a_{1}$ (TLR), and $a_{2}$ (higher-order TLR) and construct a 2nd-order polynomial. A method of constrained optimization utilizing Lagrangian multipliers is applied. Two known ISA temperatures, at SL $\left(T_{0}=288.15 \mathrm{~K}\right)$ and at $47 \mathrm{~km}(270.65 \mathrm{~K})$, are used as anchor points (constraints). The NLPAM temperature profile is then optimized by minimizing the sum-of-squares of differences between the discrete ISA and the NLPAM temperatures. The ISA temperatures are taken to be exact model values (no uncertainty). No variable weights were assigned to ISA temperatures although lower layers are indeed denser and thus contribute more to the mass of the atmosphere. It is not difficult to include specific weights in our programs if the performance measure requires it. A constrained optimization method used here is described in Appendix B. The problem with optimum approximating TLR rests for the most part in choosing the proper "figure of merit" (performance measure). Many different criteria could be used. Perhaps, the most significant criterion when 
seeking the best approximating NLPAM parabolic TLR is the conservation of tropospheric and stratospheric mass. Regardless, the presented NLPAM is still an excellent approximation and more accurate than ISOAM. However, it is only restricted to first $47 \mathrm{~km}$. Nevertheless, most of the commercial air transportation today occurs below 50,000 $\mathrm{ft}(15 \mathrm{~km})$ and NLPAM is more than sufficient.

Solving the constrained optimization model, the unknown coefficients are computed as $a_{0}=288.15$ (the first or SL constraint), $a_{1}=-5.7589736 \times 10^{-3}$, and $a_{2}=1.1460922 \times 10^{-7}$. A discriminant $D=a_{1}^{2}-4 a_{0} a_{2}=-9.8945205 \times 10^{-5}$ in the Equation 21 is negative. The optimal constrained NLPAM (Appendix B) vertical temperature profile of the ISA's troposphere and stratosphere yields $(\mathrm{H}[\mathrm{m}])$ :

$$
T(H)=288.15-5.7589736 \times 10^{-3} \cdot H+1.1460922 \times 10^{-7} \cdot H^{2}
$$

The appropriate pressure distribution from Equation 14 yields:

$$
\begin{aligned}
& \delta(H)=\delta_{0} \cdot \exp \left\{-\left(\frac{g_{0}}{R}\right) \cdot \frac{2}{\sqrt{-D}}\left[\tan ^{-1}\left(\frac{a_{1}+2 a_{2} H}{\sqrt{-D}}\right)-\tan ^{-1}\left(\frac{a_{1}}{\sqrt{-D}}\right)\right]\right\}= \\
& =\delta_{0} \cdot \exp \left\{\frac{2 \cdot g_{0}}{R \cdot \sqrt{-D}} \cdot \tan ^{-1}\left(\frac{a_{1}}{\sqrt{-D}}\right)\right\} \cdot \exp \left[-\frac{2 \cdot g_{0}}{R \cdot \sqrt{-D}} \cdot \tan ^{-1}\left(\frac{a_{1}+2 a_{2} H}{\sqrt{-D}}\right)\right]
\end{aligned}
$$

The first exponential function is just a constant for known temperature profile. One also has to remember that inverse tangent function is not unique and is bounded by $\tan ^{-1}(x) \leq|(2 n+1) \pi / 2|$, where, $-\infty<x<+\infty$. It is not difficult to show that this pressure function is monotonically decreasing with altitude. The TLR of the NLPAM is:

$$
\left[\frac{d T(H)}{d H}\right]_{N L P A M}=a_{1}+2 a_{2} H=-5.7589736 \times 10^{-3}+0.22921844 \times 10^{-6} \cdot H
$$

The NLPAM TLR is first negative, becomes zero at about $25 \mathrm{~km}$ and 216 $\mathrm{K}$, and subsequently becomes increasingly positive to hit $47 \mathrm{~km}$ at $270.65 \mathrm{~K}$ (2nd constraint is met). In the case of NLPAM, the TLR becomes zero when $H=25,119.70 \mathrm{~m}$. Density distribution as a function of geopotential height is: 


$$
\begin{aligned}
\sigma(H)=\sigma_{0} \cdot \exp \left\{\frac{2 \cdot g_{0}}{R \cdot \sqrt{-D}} \cdot \tan ^{-1}\left(\frac{a_{1}}{\sqrt{-D}}\right)\right\} \times \\
\quad \times \frac{\exp \left\{-\frac{2 \cdot g_{0}}{R \cdot \sqrt{-D}} \cdot \tan ^{-1}\left(\frac{a_{1}+2 a_{2} H}{\sqrt{-D}}\right)\right\}}{\left(1+a_{1}^{*} H+a_{2}^{*} H^{2}\right)}
\end{aligned}
$$

\section{Mass, Weight, and Scale Heights of Atmosphere}

The mass (not to be confused with previous symbol $\mathrm{M}$ for molecular weight or Mach number) of the ISA atmosphere for the spherical Earth of uniform mass-density and zero (MSL) elevation can be calculated from (Daidzic, 2015):

$$
M(z)=\iiint_{V(z)} \rho(z) d V=4 \pi R_{0}^{2} \rho_{0} \int_{0}^{z} \sigma(z)\left(1+\frac{z}{R_{0}}\right)^{2} d z \quad 0 \leq z<\infty
$$

Similarly, the weight of an atmospheric layer by knowing the air density distribution and the change of gravitational acceleration is (Daidzic, 2015):

$W(z)=\iiint_{V(z)} \rho(z) g(z) d V=4 \pi R_{0}^{2} g_{0} \rho_{0} \int_{0}^{z} \sigma(z) d z$

These integrals can be evaluated analytically or numerically. A good historical account of atmospheric mass estimates is given in Trenberth (1981). Davies (2003) reports the value of atmospheric mass of approximately $5.2 \times 10^{18} \mathrm{~kg}$. Recently, Trenberth and Smith (2005) reported the dry mass of the atmosphere to be $(5.1352 \pm 0.0003) \times 10^{18} \mathrm{~kg}$. The authors perform integration starting from the globally-averaged surface topography elevation using the zonally-averaged surface pressure. More recently, Daidzic (2015) calculated the mass and weight of ISA up to $86 \mathrm{~km}$. Daidzic estimated the mass of ISA homosphere as $5.294480 \times 10^{18} \mathrm{~kg}$ and the weight is $5.180137 \times 10^{19} \mathrm{~N}$. However, those computations overestimated the Earth's atmospheric mass somewhat as the lower bound was SL and not average surface level (about $237 \mathrm{~m}$ ). Vertical change of gravitational acceleration according to Newtonian law of universal gravitation was included in weight calculations, but gravitational anomalies were excluded.

The mass of dry ISOAM assuming it extends from SL to infinity and after replacing the orthometric with the geopotential altitude in Equation 27, yields: 
$M_{0}=4 \pi R_{0}^{2} \rho_{S L} \sigma_{0} \int_{0}^{\infty}\left(1+\frac{H}{R_{0}-H}\right)^{4} \exp \left(-\kappa^{*} H\right) d H<\infty \quad H \neq R_{0}$

The spherical Earth's superficial area with the mean radius of $6,371 \mathrm{~km}$ (Stacey \& Davis, 2008) is $S_{0}=4 \pi R_{0}^{2} \approx 5.1 \times 10^{14} \mathrm{~m}^{2}$. As $H \rightarrow R_{0}$, the above integral will indeed converge rapidly as the exponential function in integrand decreases to zero much faster than the 4th-order polynomial increases. Thus in the limit one can write:

$$
\begin{aligned}
& M_{0} \approx\left(S_{0} \rho_{S L} \sigma_{0}\right) \int_{0}^{\infty}\left(1+4 \frac{H}{R_{0}}\right) \exp \left(-\kappa^{*} H\right) d H= \\
& \quad=\left(S_{0} \rho_{0}\right) \cdot\left(\frac{1}{\kappa^{*}}+\frac{1}{R_{0}} \frac{4}{\kappa^{* 2}}\right)=\frac{S_{0} \cdot p_{0}}{g_{0}} \cdot\left(1+\frac{4 \cdot H^{*}}{R_{0}}\right)
\end{aligned}
$$

The magnitude of the second term is: $\left(4 \cdot H^{*} / R_{0}\right) \approx 0.0051$. In a good approximation the second term in Equation 30 can thus be neglected and the mass of the atmosphere simply becomes:

$$
M_{0} \approx \frac{S_{0} \cdot \rho_{S L} \cdot \sigma_{0}}{\kappa^{*}}=\frac{S_{0} \cdot p_{0}}{g_{0}}
$$

This agrees well with the theoretical consideration by Trenberth and Smith (2005) in which the mass of the atmosphere is directly proportional to the zonalaveraged (oblate spheroid) surface pressure. The approximate weight is estimated directly from Equation 31. Replacing known ISA SL values into Equation 31, one obtains $5.270126 \times 10^{18} \mathrm{~kg}$ and $5.296761 \times 10^{18} \mathrm{~kg}$ (Equation 30) respectively. Using the averaged measured dry air surface pressure of $983.05 \mathrm{hPa}$ (Trenberth \& Smith, 2005) accounting for non-zero average topography elevation, the atmospheric mass becomes $5.113430 \times 10^{18} \mathrm{~kg}$ according to Equation 31 and $5.13927183 \times 10^{18} \mathrm{~kg}$ when the second-term from Equation 30 is included.

Using the simple ISOAM, a fraction-mass scale-height can be easily estimated. The approximate mass of the ISOAM atmosphere with $H<<R_{0}$, is:

$$
M(H) \approx\left(S_{0} \rho_{S L} \sigma_{0}\right) \int_{0}^{H} \exp \left(-\kappa^{*} H\right) d H=M_{0}\left[1-\exp \left(-\kappa^{*} H\right)\right]
$$


The scale height of the 275K ISOAM's arbitrary fractional mass, using the ISA SL pressure $\left(\sigma_{0}=\delta_{0} T_{S L} / T_{0}=1.047817\right)$, is estimated from:

$$
H=-\frac{R T_{0}}{g_{0}} \cdot \ln \left[1-\frac{M(H)}{M_{0}}\right]=-H^{*} \cdot \ln (1-f) \quad f=\frac{M(H)}{M_{0}}
$$

where: $M_{0}=5.296761 \times 10^{18} \mathrm{~kg} \quad T_{0}=275 \mathrm{~K} \quad H^{*}=8,049.60 \mathrm{~m}$.

In the case of NLPAM, the mass of any particular atmospheric layer becomes, by utilizing Equations 5 and 27:

$$
M=4 \pi R_{0}^{2} \rho_{0} \int_{z_{1}}^{z_{2}} \sigma(z)\left(1+\frac{z}{R_{0}}\right)^{2} d z=S_{0} \rho_{0} \int_{H_{1}}^{H_{2}}\left(1+\frac{H}{R_{0}-H}\right)^{4} \sigma(H) d H
$$

Substituting this expression for density distribution for an arbitrary layer from Equation 26 into Equation $34\left(M_{1}=1.6999321 \times 10^{13}\right)$, yields:

$$
\begin{aligned}
& M(H)=S_{0} \rho_{S L} \sigma_{0} \cdot \exp \left[\left(\frac{g_{0}}{R}\right) \cdot \frac{2}{\sqrt{-D}} \cdot \tan ^{-1}\left(\frac{a_{1}}{\sqrt{-D}}\right)\right] \cdot I(H)=M_{1} \cdot I(H) \\
& I(H) \approx \int_{H_{1}}^{H_{2}} \frac{\left(1+H / R_{0}\right)^{4}}{\left(1+a_{1}^{*} H+a_{2}^{*} H^{2}\right)} \exp \left[-\left(\frac{g_{0}}{R}\right) \cdot \frac{2}{\sqrt{-D}} \cdot \tan ^{-1}\left(\frac{a_{1}+2 a_{2} H}{\sqrt{-D}}\right)\right] d H
\end{aligned}
$$

This complex-looking integral can be approximately evaluated with the power-series expansion. However, since the integrand is a known function, a powerful numerical 8-point Gaussian-Legendre quadrature (Carnahan et al., 1969; Chapra \& Canale, 2006; Conte \& de Boor, 1986; Demidovich \& Maron, 1987; Press et al., 1992; Ralston \& Rabinowitz 1978) utilizing Legendre orthogonal polynomials (Lebedev, 1972) is employed to calculate masses.

Masses of individual atmospheric layers in ISOAM and NLPAM atmospheres and the total mass was evaluated numerically and analytically when possible. A ForTRAN 95 (Lahey Computer Systems, Inc., Incline Village, NV, USA) optimizing compiler with IMSL (Visual Numerics, Inc., Houston, TX, USA) and MATLAB ${ }^{\circledR}$ (The Mathworks, Inc., Natick, MA, USA) high-level programing languages were utilized to design in-house programs to calculate the integrals given by Equations 29 (ISOAM) and 36 (NLPAM) mostly using Gauss- 
Legendre numerical integration method highlighted in Appendix C. Additionally, trapezoidal, recursive adaptive Simpson, recursive adaptive Lobatto, and GaussCronrod quadrature methods (Press et al., 1992) have also been used with MATLAB ${ }^{\circledR}$ for verification with minimum required accuracy of $10^{-6}$. Many tests of various numerical integration methods were performed yielding satisfactory results although some MATLAB $^{\circledR}$ intrinsic integrators were overly sensitive to integration limits. In-house designed integrators typically performed better.

\section{Discussion of Results}

Temperature profiles of ISA, ISOAM, and NLPAM up to about $50 \mathrm{~km}$ orthometric height are shown in Figure 1. While ISA and ISOAM extend up to 86 orthometric km, NLPAM extends only up to 47 geopotential $\mathrm{km}$. The temperature at which the TLR is zero is $215.8 \mathrm{~K}$ or about $-57^{\circ} \mathrm{C}$ for NLPAM. This temperature profile indicates almost identical TLR at SL $\left(-1.755^{\circ} \mathrm{C} / 1000\right.$ feet $)$ compared to ISA's $-1.98^{\circ} \mathrm{C} / 1000$ feet which progressively shallows out and then becomes positive from about $25 \mathrm{~km}$ upwards.

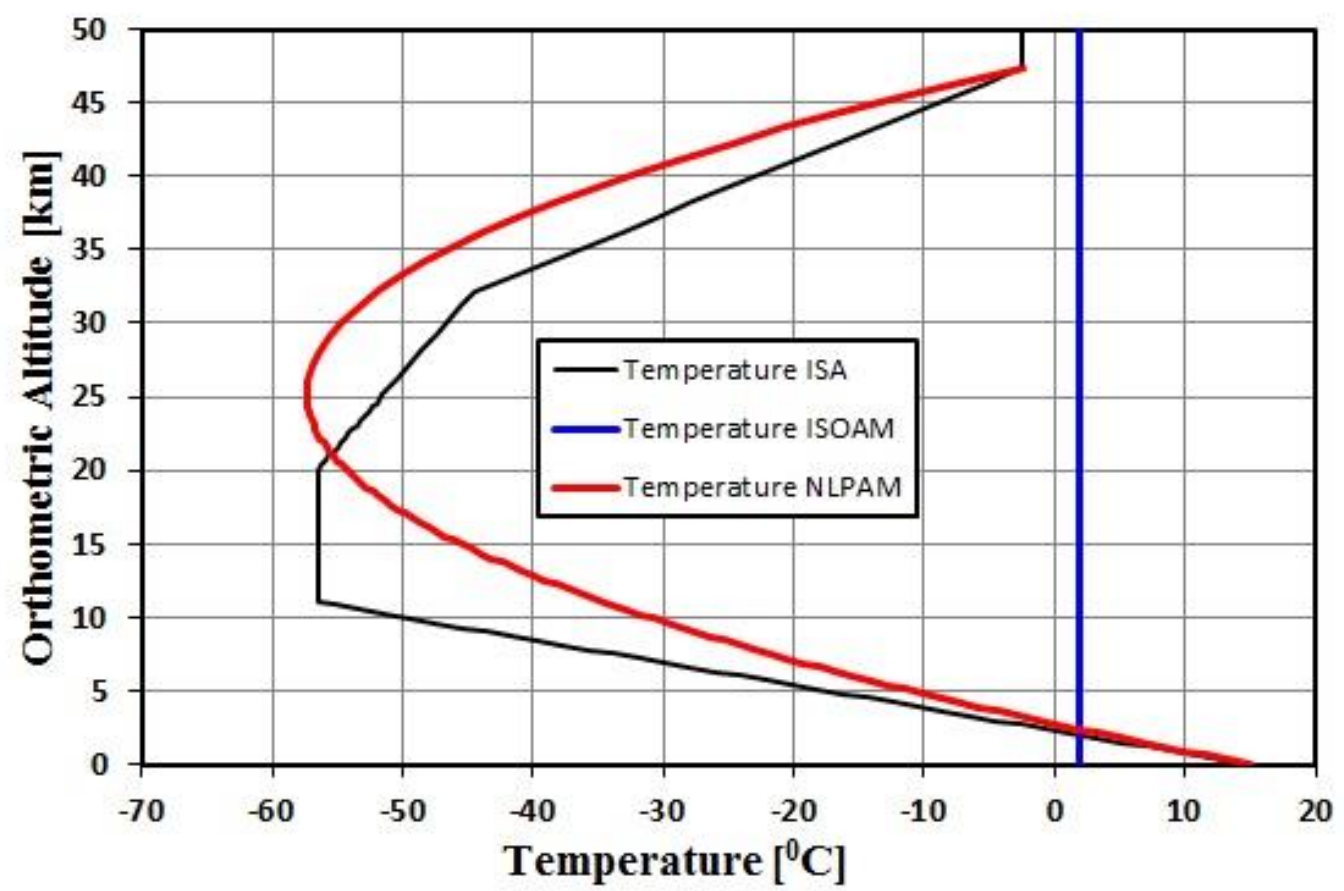

Figure 1. Vertical temperature profiles for ISA, ISOAM and NLPAM versus orthometric altitude. 
Temperature lapse rates for ISA, ISOAM, and NLPAM atmospheres are shown in Figure 2. The optimum ISOAM constant temperature was estimated (Appendix A) and rounded to an integer value of $275 \mathrm{~K}\left(+2^{\circ} \mathrm{C}\right)$. The TLR for ISOAM is, of course, zero. The ISA's TLRs jump across base layers. The NLPAM TLR changes smoothly from negative in the lower layers (troposphere and tropopause), becomes roughly isothermal around $25 \mathrm{~km}$, (ISA is isothermal from 11 to $20 \mathrm{~km}$ ) and then positive in upper stratosphere. Instead of four different TLRs for ISA, there is one TLR in both ISOAM and NLPAM. A TLR based on the third-order polynomial could be used to also model mesosphere.

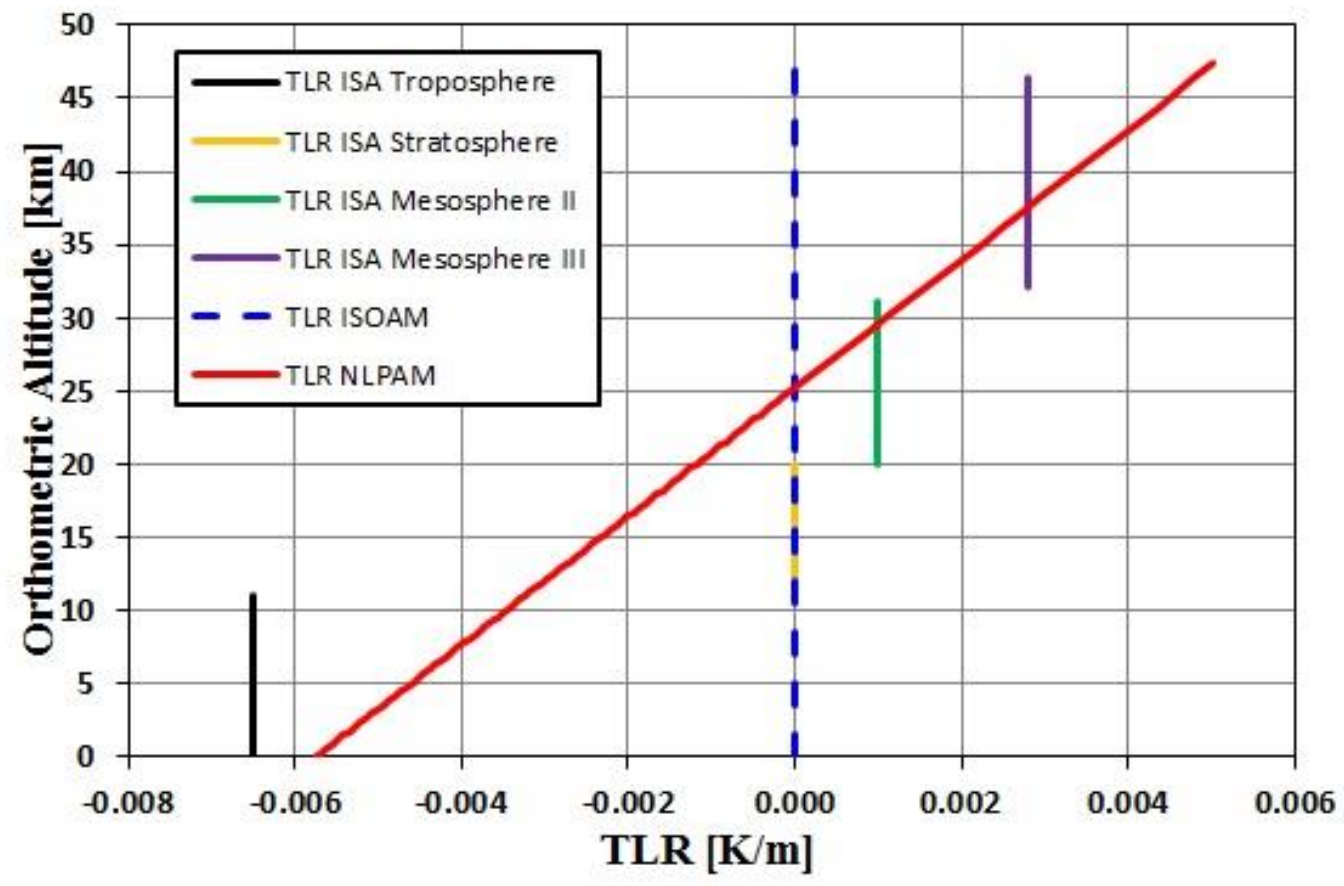

Figure 2. TLRs for ISA, ISOAM and NLPAM versus orthometric altitude.

The comparison between ISA's and ISOAM's non-dimensional pressure and density distribution up to $86 \mathrm{~km} \mathrm{MSL}$ is shown in Figure 3. It seems the difference between respective pressures and densities is excessive at high altitudes but one must bear in mind that most of the atmospheric mass is distributed in lower altitudes. It is thus more important to approximate ISA well at low altitudes which is exactly what the minimum sum of density differences squares is achieving here constrained by the ideal-gas law and ISA's surface pressure at the SL. The results for ISA and ISOAM zoomed for the first $47 \mathrm{~km}$ are shown in 
Figure 4. The approximation is particularly good up to $20 \mathrm{~km}$. Many different isothermal atmospheres can be designed based on the specific merit functions.

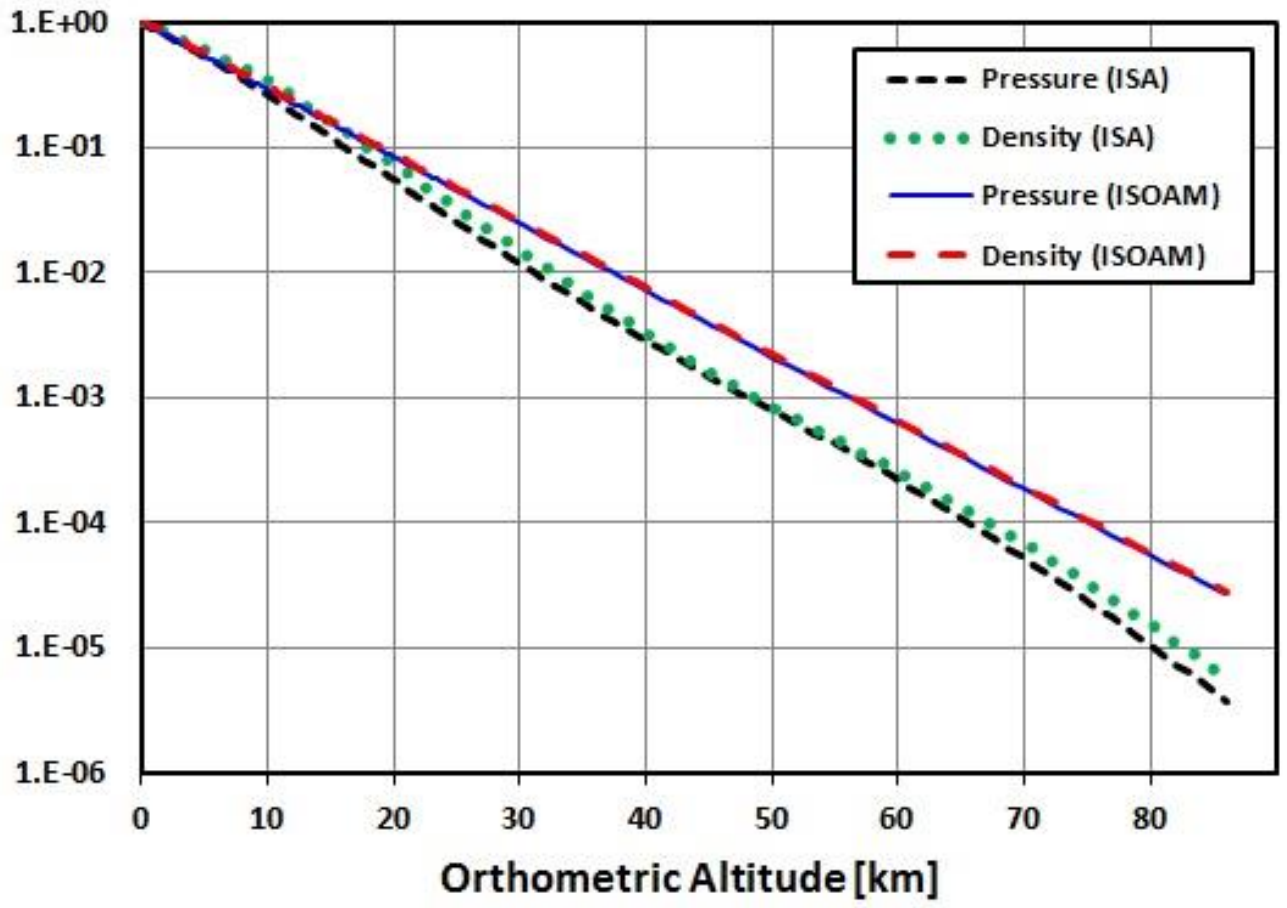

Figure 3. Comparison of non-dimensional pressures and densities for ISA and the fictional isothermal $275 \mathrm{~K}$ ISOAM up to 86 orthometric $\mathrm{km}$.

A comparison of ISA's and NLPAM's vertical pressure and density distributions are shown in Figure 5. Clearly, since the NLPAM's TLR approximates meandering ISA's TLR well up to $47 \mathrm{~km}$, it is to be expected that the vertical pressure and density distributions will be satisfactory using the vertical temperature profile expressed by Equation 23. The benefit of having only one, though arguably more complex, TLR expression instead of four different in ISA is clear. Pressure calculations using Equation 24 are only marginally more complicated than any of individual ISA's expressions.

The mass of the ISOAM atmosphere can be quickly estimated from the surface air pressure by using Equations 30 or 31 . The aerostatic balance implies that the atmospheric pressure at given height is equivalent to the weight of the column of air above that particular height per unit surface area. The total weight of the atmosphere then is surface pressure multiplied by the Earth's surface area. This is reasonable as long as the Earth is assumed to be spherically symmetric and 
the surface pressure uniform over entire surface. If one assumes the average dryair surface pressure of $983.05 \mathrm{mbar}$ (or $\mathrm{hPa}$ ) according to global measurements (Trenberth \& Smith, 2005), the weight of the atmosphere is $5.014189 \times 10^{19} \mathrm{~N}$.

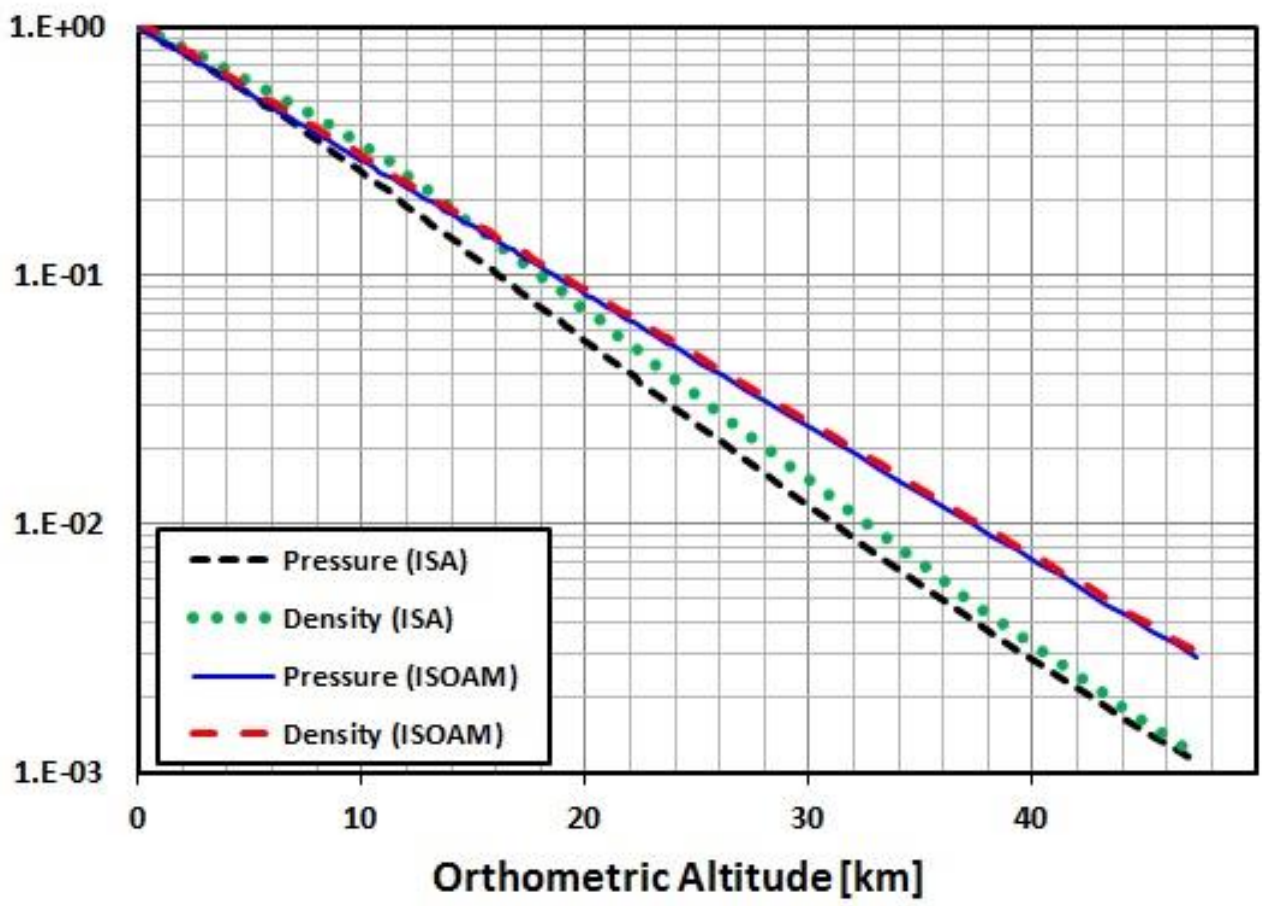

Figure 4. Comparison of non-dimensional pressures and densities for ISA and the fictional isothermal $275 \mathrm{~K}$ atmosphere up to 47 geopotential $\mathrm{km}$ (ISOAM).

At SL standard acceleration this weight corresponds to a dry-air mass of $5.11305 \times 10^{18} \mathrm{~kg}$ (Equation 31). Using Equation 30 a slightly better estimate is achieved of $5.138891 \times 10^{18} \mathrm{~kg}$. This is very close to recent estimates given by Trenberth and Smith (2005) and not far from the ISA mass calculations by Daidzic (2015). It is also in decent agreement with the amount of $5.28 \times 10^{18} \mathrm{~kg}$ by Stacey and Davis (2008).

The mass computations for NLPAM is bit more complicated and has been obtained numerically. The dry-air atmospheric mass by layer and the total for all three models (ISA, ISOAM, and NLPAM) are summarized in Tables 3 and 4 using various quadrature methods. Judging from Table 3 it is clear that the difference between ISA and ISOAM is very small in terms of the total mass (less than $0.05 \%$ ). Additionally, the difference between analytical and numerical 
ISOAM predictions is negligible. The NLPAM is not valid in mesosphere, but as calculated by Daidzic (2015) almost $99.9 \%$ of atmosphere is already contained below $47 \mathrm{~km}$ and so the total NLPAM mass is not significantly smaller than the ISA total. As shown in Table 4, the difference between the ISA and NLPAM masses is relatively small even for each particular ISA layer.

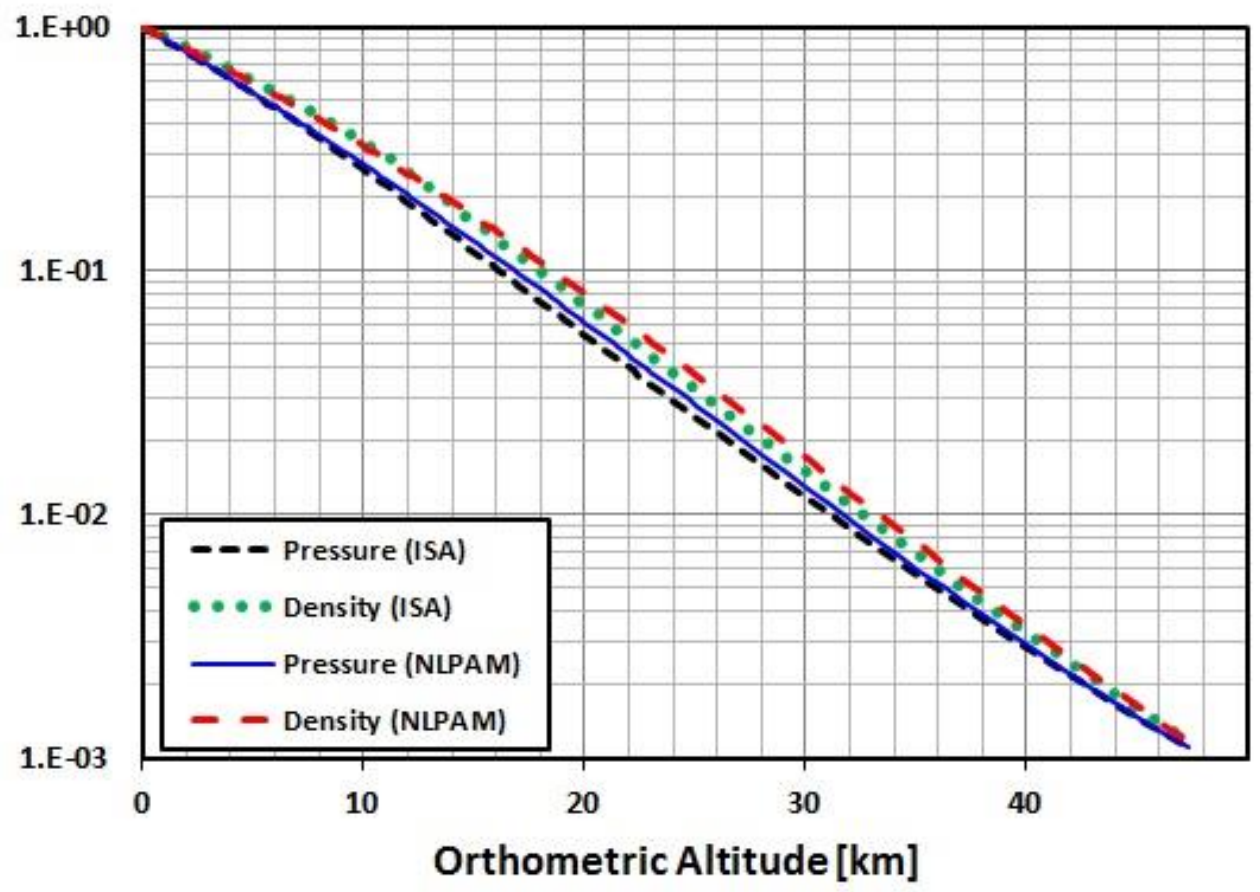

Figure 5. Comparison of non-dimensional pressures and densities for ISA and the fictional parabolic atmosphere (NLPAM) up to 47 geopotential $\mathrm{km}$.

The mass of the Earth's atmosphere above $86 \mathrm{~km}$ is negligible compared to the total sum over lower layers. Specific ISOAM and NLPAM masses are depicted in Figures 6 and 7. Essentially, specific masses are integrands in mass integrals. Decrease of the specific mass is linear (ISOAM) or almost linear (NLPAM) in logarithmic scale. Masses can be quickly estimated from the average values using Figures 6 and 7.

The fractional atmospheric masses for three atmospheric models and their respective scale heights are summarized in Table 5. Calculated scale-heights are accurate within about $\pm 1 \%$ (about $\pm 50 \mathrm{~m}$ in ISA troposphere) and the values were not interpolated, but taken as closest to tabulated fractional mass (Daidzic, 2015). The values for ISOAM fractional-mass scale-heights are calculated using 
Equation 33. A change of ISOAM mass scale heights with the mass fraction is shown in Figure 8. The slope of the scale height at SL is equal to ISOAM scale height from the following expression (using Equation 33):

$$
\frac{d H}{d f}=\frac{H^{*}}{(1-f)} \quad f \in[0,1)
$$

The values of scale heights for NLPAM are obtained from integrated mass using Equation 34 and method highlighted in Appendix C.

Table 3

Masses of Layers for ISA and ISOAM's analytical and numerical Computations

\begin{tabular}{lcccc}
\hline \multicolumn{1}{c}{ ISA Level } & $\begin{array}{c}\text { Mass [kg] } \\
\text { ISA }\end{array}$ & $\begin{array}{c}\text { Mass [kg] } \\
\text { ISOAM } \\
\text { (Eq. 29) }\end{array}$ & $\begin{array}{c}\text { Mass [kg] } \\
\text { ISOAM } \\
\text { (8-point G-L) }\end{array}$ & $\begin{array}{c}\text { Mass [kg] } \\
\text { ISOAM } \\
\text { (Matlab) }\end{array}$ \\
\hline Troposphere & $4.104397 \mathrm{E}+18$ & $3.946136 \mathrm{E}+18$ & $3.9368841 \mathrm{E}+18$ & $3.9368818 \mathrm{E}+18$ \\
Tropopause & $9.005369 \mathrm{E}+17$ & $9.090914 \mathrm{E}+17$ & $9.1290622 \mathrm{E}+17$ & $9.1290540 \mathrm{E}+17$ \\
Stratosphere II & $2.432901 \mathrm{E}+17$ & $3.420996 \mathrm{E}+17$ & $3.4568045 \mathrm{E}+17$ & $3.4568003 \mathrm{E}+17$ \\
Stratosphere III & $4.030482 \mathrm{E}+16$ & $8.400837 \mathrm{E}+16$ & $8.5572534 \mathrm{E}+16$ & $8.5572396 \mathrm{E}+16$ \\
Stratopause & $2.358320 \mathrm{E}+15$ & $6.040763 \mathrm{E}+15$ & $6.1982633 \mathrm{E}+15$ & $6.1982512 \mathrm{E}+15$ \\
Mesosphere II & $3.396125 \mathrm{E}+15$ & $8.602831 \mathrm{E}+15$ & $8.8742306 \mathrm{E}+15$ & $8.8742108 \mathrm{E}+15$ \\
Mesosphere III & $1.960204 \mathrm{E}+14$ & $6.423663 \mathrm{E}+14$ & $6.7058114 \mathrm{E}+14$ & $6.7057925 \mathrm{E}+14$ \\
\hline \multicolumn{1}{c}{ TOTAL } & $5.294480 \mathrm{E}+18$ & $5.296621 \mathrm{E}+18$ & $5.2967863 \mathrm{E}+18$ & $5.2967827 \mathrm{E}+18$ \\
\hline
\end{tabular}

Table 4

Masses of ISA Layers for ISA and NLPAM

\begin{tabular}{lccc}
\hline \multicolumn{1}{c}{ ISA Level } & $\begin{array}{c}\text { Mass [kg] } \\
\text { ISA }\end{array}$ & $\begin{array}{c}\text { Mass [kg] } \\
\text { NLPAM } \\
\text { (8-point G-L) }\end{array}$ & $\begin{array}{c}\text { Mass [kg] } \\
\text { NLPAM } \\
\text { (Matlab) }\end{array}$ \\
\hline Troposphere & $4.104397 \mathrm{E}+18$ & $4.0370412 \mathrm{E}+18$ & $4.09099403 \mathrm{E}+18$ \\
Tropopause (SS I) & $9.005369 \mathrm{E}+17$ & $9.3145274 \mathrm{E}+17$ & $9.38094877 \mathrm{E}+17$ \\
Stratosphere II & $2.432901 \mathrm{E}+17$ & $2.7670765 \mathrm{E}+17$ & $2.75927071 \mathrm{E}+17$ \\
Stratosphere III & $4.030482 \mathrm{E}+16$ & $4.3888902 \mathrm{E}+16$ & $4.20237335 \mathrm{E}+16$ \\
Stratopause (MS I) & $2.358320 \mathrm{E}+15$ & NA & NA \\
Mesosphere II & $3.396125 \mathrm{E}+15$ & NA & NA \\
Mesosphere III & $1.960204 \mathrm{E}+14$ & NA & NA \\
\hline \multicolumn{1}{c}{ TOTAL } & $5.294480 \mathrm{E}+18$ & $5.2890905 \mathrm{E}+18$ & $5.3470397 \mathrm{E}+18$ \\
\hline
\end{tabular}




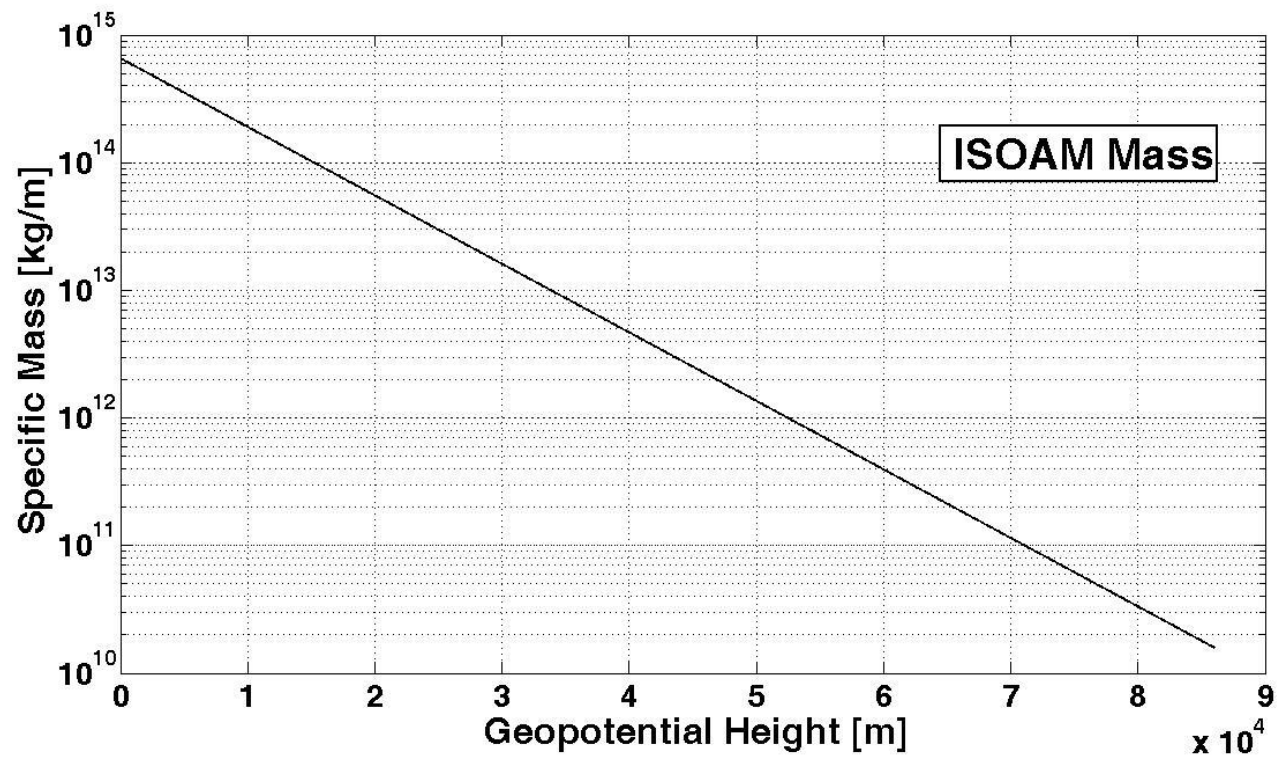

Figure 6. ISOAM specific mass as a function of geopotential height.

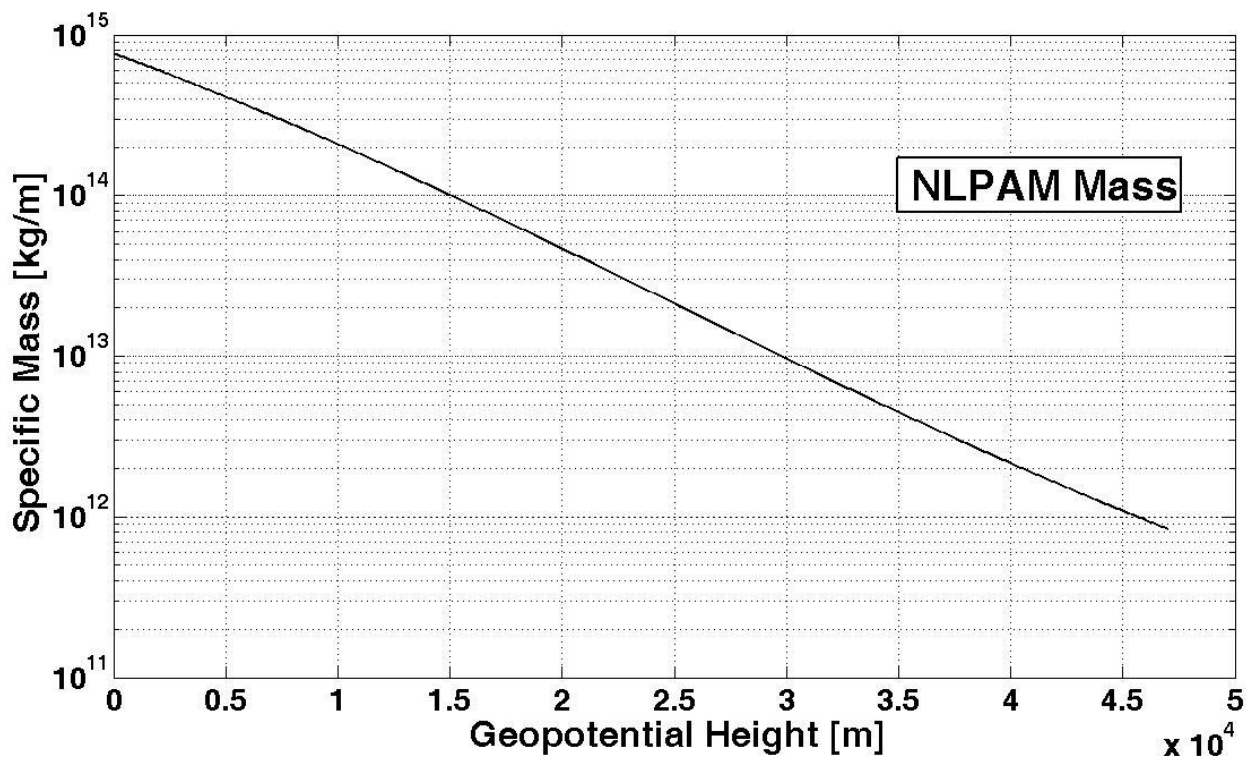

Figure 7. NLPAM specific mass as a function of geopotential height.

An example comparing atmospheric parameters for ISA, ISOAM and NLPAM at a given altitude/height will be in order now. A comparison of 
dimensional and non-dimensional atmospheric parameters at a geopotential height of $40 \mathrm{~km}$ is summarized in Table 6. The NLPAM temperature at $40 \mathrm{~km}$ is colder by about $10^{\circ} \mathrm{C}$, while the difference in pressure and density are less than $2.6 \%$ and $6.5 \%$ respectively.

Table 5

Mass fraction as Functions of Orthometric Scale Heights for ISA, ISOAM, and NLPAM

\begin{tabular}{cccc}
\hline Mass fraction & ISA [m] & ISOAM [m] & NLPAM [m] \\
\hline $50 \%$ & 5,405 & $5,579.56$ & $5,557.50$ \\
$75 \%$ & 10,216 & $11,159.11$ & $10,615.00$ \\
$90 \%$ & 16,040 & $18,534.89$ & $16,800.00$ \\
$95 \%$ & 20,315 & $24,114.44$ & $21,200.00$ \\
$99 \%$ & 30,899 & $37,069.77$ & $30,922.00$ \\
$99.9 \%$ & 47,857 & $55,604.66$ & $42,185.00$ \\
\hline
\end{tabular}

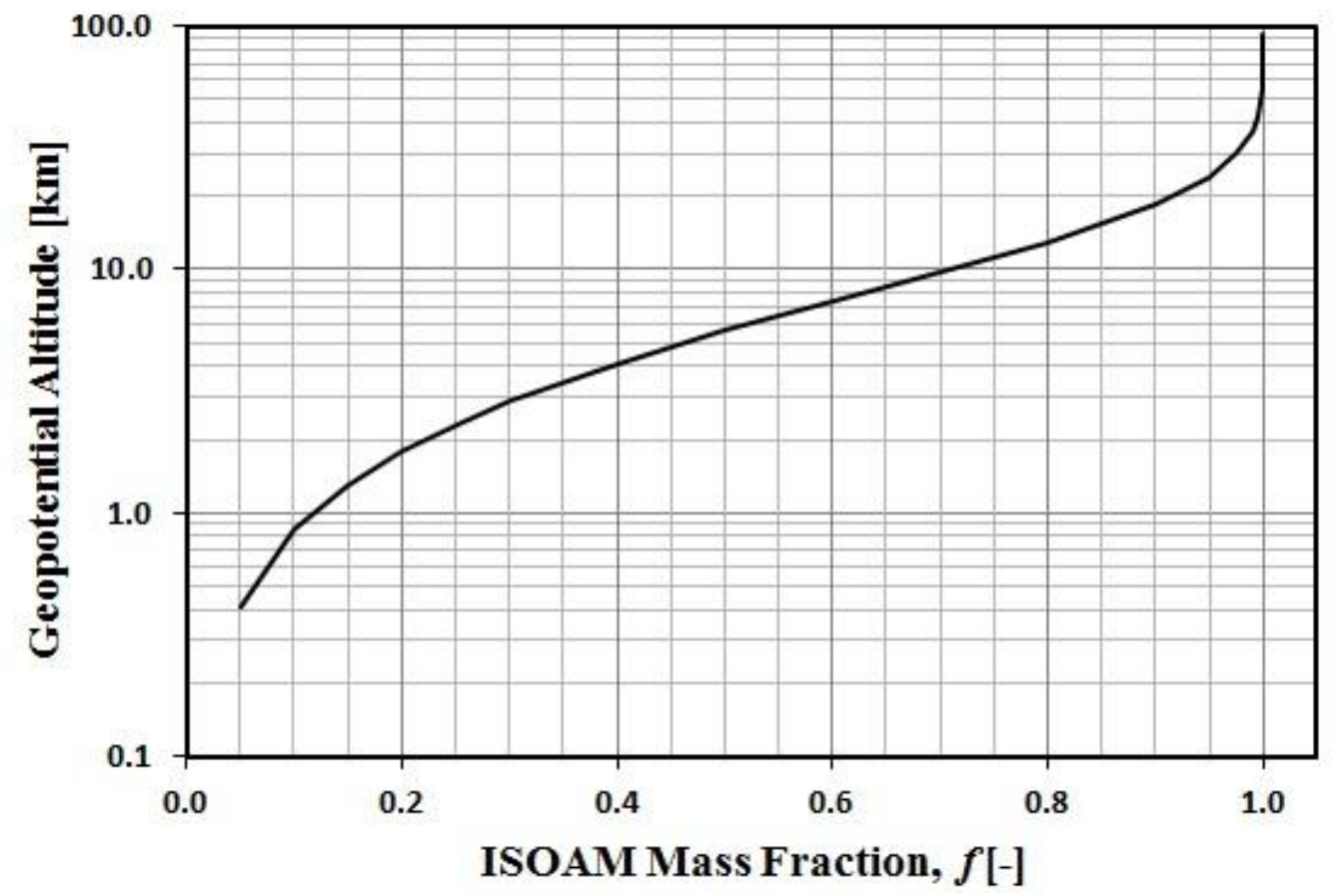

Figure 8. Scale heights versus the 275 K ISOAM mass fractions. 
On the other hand, the ISOAM temperature is quite warmer and the pressure and density are more than twice as large. However, considering that the pressure and density are more than two orders-of-magnitude lower compared to SL conditions, it does not influence the total mass much. The ISOAM will overestimate drag and lift calculations at these heights which is acceptable in the first approximation.

Table 6

Comparison of ISA, ISOAM, and NLPAM Atmospheric Parameters at $40 \mathrm{~km}$ Geopotential Height

\begin{tabular}{cccc}
\hline & ISA & ISOAM & NLPAM \\
\hline $\mathrm{H}[\mathrm{m}]$ & 40,000 & 40,000 & 40,000 \\
$\mathrm{z}[\mathrm{m}](\mathrm{Eq} .5)$ & 40,253 & 40,253 & 40,253 \\
$T\left[\mathrm{~K} /{ }^{0} \mathrm{C}\right]$ & $251.05 /(-22.10)$ & $275 /(+1.85)$ & $241.21 /(-31.94)$ \\
$\theta[-]$ & 0.8712476 & 0.954364047 & 0.8370948 \\
$p[\mathrm{~Pa}]$ & 277.52155 & 704.08317 & 285.07077 \\
$\delta[-]$ & $2.738925 \mathrm{E}-03$ & $6.9487606 \mathrm{E}-03$ & $2.8134298 \mathrm{E}-03$ \\
$\rho\left[\mathrm{kg} / \mathrm{m}^{3}\right]$ & $3.8510095 \mathrm{E}-03$ & $8.9192651 \mathrm{E}-03$ & $4.1171580 \mathrm{E}-03$ \\
$\sigma[-]$ & $3.143681 \mathrm{E}-03$ & $7.2810377 \mathrm{E}-03$ & $3.3609453 \mathrm{E}-03$ \\
\hline
\end{tabular}

Future work on this subject will focus on introducing new methods of atmospheric mass computations including Earth's real shape (Geoid), terrain elevation, curved geometry aero/hydro-static equation, and incorporating gravitational anomalies. The shape of the Earth and the gravitational magnitudes will be represented in a series of spherical tesseral harmonics.

\section{Conclusions}

Both, a new global isothermal temperature atmospheric model and a nonlinear parabolic-temperature atmospheric models of the ISA are introduced. Constrained optimization techniques in conjunction with the least-square-root approximations were utilized to design best-fit isothermal models for ISA pressure and density changes up to 47 geopotential $\mathrm{km}$ for NLPAM and 86 orthometric $\mathrm{km}$ for ISOAM. The mass of the dry-air atmosphere and the relevant fractional-mass scale heights have been estimated utilizing accurate and sophisticated Gauss-Legendre numerical quadrature for both ISOAM and NLPAM. Both, ISOAM and NLPAM represent viable alternatives to ISA in many 
practical applications. A true benefit of ISOAM and NLPAM is that only single expressions for temperature, pressure, and density are used instead of many different expressions in ISA formulation. A parabolic NLPAM is especially accurate in substituting ISA up to 47 geopotential km. Fractional mass scaleheights have been calculated for both ISAOM and NLPAM and compared to ISA values. As expected, the agreement is especially good between ISA and NLPAM. It is straightforward to extend the notion of nonlinear temperature distribution and utilize higher-order polynomials describing vertical temperature profile to higher altitudes in mesosphere and this will be done in a subsequent publication. ISA, ISOAM, and NLPAM overestimate the mass of the real dry atmosphere by small amount as the lower limit of integration is taken as SL in ISA models and not as an average topography height of about $237 \mathrm{~m}$ in real atmospheric mass estimates. Globally and temporally averaged surface pressure measurements of $983.05 \mathrm{hPa}$ are about $30 \mathrm{hPa}$ lower than the adopted standard SL ISA pressure and about 28 $\mathrm{hPa}$ lower than globally measured average SL pressure of $1011.00 \mathrm{hPa}$.

\section{Acknowledgment}

The author would like to express his gratitude to anonymous reviewers for making useful suggestions and to the Editorial Staff of the IJAAA for their assistance in conforming to manuscript formatting guidelines and in proof-reading the text. 


\section{Author Bios}

Dr. Nihad E. Daidzic is president of AAR Aerospace Consulting, L.L.C. He is also a full professor of Aviation, adjunct professor of Mechanical Engineering, and research graduate faculty at Minnesota State University, Mankato. His Ph.D. is in fluid mechanics and Sc.D. in mechanical engineering. He was formerly a staff scientist at the National Center for Microgravity Research and the National Center for Space Exploration and Research at NASA Glenn Research Center in Cleveland, $\mathrm{OH}$. He has also held various faculty appointments at Vanderbilt University, University of Kansas, and Kent State University. His current research interest is in theoretical, experimental, and computational fluid dynamics, microand nano-fluidics, aircraft stability, control, and performance, mechanics of flight, piloting techniques, and aerospace propulsion. Dr. Daidzic is ATP and "Gold Seal" CFII/MEI/CFIG with flight experience in airplanes, helicopters, and gliders. 


\section{Appendix A}

Nonlinear regression for global isothermal model

A regression (best-fit) of the measured or model data with an exponential function yields:

$$
f\left(a_{0}, a_{1} ; x\right)=a_{0} \exp \left(-a_{1} x\right)
$$

This exponential model has two degrees-of-freedom (coefficients: $a_{0}, a_{1}$ ). A task of classical unconstrained regression analysis is to find the two unknown coefficients which will minimize the performance measure (most often the residual sum of squared differences between the observed and model data):

$$
S_{r}=\sum_{i=1}^{N}\left[y_{i}-f\left(a_{0}, a_{1} ; x_{i}\right)\right]^{2}=\|\mathbf{y}-f(\mathbf{a})\|_{2}
$$

However, Equation A1 is nonlinear in respect to two unknown coefficients. To treat this as a part of linear regression analysis and estimate optimum coefficients, the exponential form in Equation A1 needs to be first linearized by using the logarithm:

$\ln y=\ln a_{0}-a_{1} \cdot x \Rightarrow Y=A_{0}-A_{1} \cdot x$

However, this procedure should normally be avoided unless the errors are log-normally distributed. Error structure (additive, proportional and multiplicative error) must be considered before making linearizing transformations (Seber \& Wild, 1989). Otherwise, from here the process is trivial and the theory of linear regression (normal equations) by a linear (straight) curve is used with details provided in many classical books on numerical analysis (e.g., Carnahan et al., 1969; Chapra \& Canale, 2006; Conte \& de Boor, 1986; Press et al., 1992). Also the polynomial approximation to be dealt with later belongs to the general theory of linear regression as the polynomial is linear in unknown coefficients irrespective of the possible wild nonlinearities in the basis functions.

On the other hand, the problem that is solved in this work has only one degree-of-freedom and is nonlinear since the SL density is constrained by the ideal-gas law and dependent on chosen SL pressure (ISA SL pressure of 1013.25 $\mathrm{hPa}$ ) and unknown yet to be found isothermal temperature. The scale factor in 
exponent which is the inverse of the atmospheric scale height is also a function of unknown uniform temperature. Thus we may write:

$$
\rho\left(a_{0}, a_{1} ; H\right)=a_{0}\left(T_{0}\right) \cdot \exp \left[-a_{1}\left(T_{0}\right) \cdot H\right] \quad a_{0}=\rho_{0}=\frac{p_{0}}{R T_{0}} \quad a_{1}=\kappa^{*}=\frac{g_{0}}{R T_{0}}
$$

The sum of squares that needs to be minimized is:

$S_{r}\left(a_{0}, a_{1}\right)=\sum_{i=1}^{N}\left[\rho_{I S A}\left(H_{i}\right)-\rho_{I S O A M}\left(a_{0}, a_{1} ; H_{i}\right)\right]^{2}$

The two coefficients are dependent and the model is thus nonlinear. For example, a Gauss-Newton (G-N) method of successive approximations (iterations) can be used for two or more variables (Chapra \& Canale, 2006; Press et al., 1992; Seber \& Wild, 1989). As usual, a merit function (typically, $\mathrm{L}^{2}$ norm) is minimized which is often just a familiar sum of squares:

$$
\frac{\partial S_{r}}{\partial a_{k}}=0=2\left\{\sum_{i=1}^{N}\left[y_{i}-f\left(a_{k} ; x_{i}\right)\right] \frac{\partial f\left(a_{k} ; x_{i}\right)}{\partial a_{k}}\right\} \quad k=1,2, \ldots, K<N
$$

A resulting system of equations is nonlinear and is solved in an iterative manner using the G-N method. The linear (first-order) term in Taylor expansion of approximating model equation is used:

$f\left(a_{k}^{j+1} ; x_{i}\right) \approx f\left(a_{k}^{j} ; x_{i}\right)+\sum_{k=1}^{K} \frac{\partial f\left(a_{k}^{j} ; x_{i}\right)}{\partial a_{k}}\left(a_{k}^{j+1}-a_{k}^{j}\right)=f\left(a_{k}^{j} ; x_{i}\right)+\sum_{k=1}^{K} J_{i k} \Delta a_{k}$

where: $J_{i k}=\frac{\partial f\left(a_{k}^{j} ; x_{i}\right)}{\partial a_{k}}$.

An initial guess $a_{k}^{0}$ is required for every unknown coefficient (vector of unknowns) to start the process and thus the convergence is not ensured. The Jacobian matrix is evaluated at each iteration step. A system (Equation A7) reduces to normal equations of linear regression which must be solved at each successive approximation (step):

$$
\left(\mathbf{J}^{\mathbf{T}} \mathbf{J}\right) \Delta a=\mathbf{J}^{\mathbf{T}} \Delta y \quad \Rightarrow \quad \Delta a=\left(\mathbf{J}^{\mathbf{T}} \mathbf{J}\right)^{-1} \mathbf{J}^{\mathbf{T}} \Delta y \quad \Delta y_{i}=y_{i}-f\left(a_{k}^{j} ; x_{i}\right)
$$


Updated solutions are calculated (shifted) based on the just calculated (positive and/or negative) increments $a_{k}^{j+1}=a_{k}^{j}+\Delta a$ and the process is continued until the relative error falls below a prescribed ones. However, the convergence will depend on the good choice of the initial values and is not ensured. Traditional methods, such as, Gauss-Jordan elimination, LU decomposition, Cholesky decomposition, etc., may work in most instances for simpler systems. However, the normal equations are very often close to singular (ill-conditioned) and very susceptible to round-off errors (Press et al., 1992). Advanced algorithms are needed such as QR decomposition and SVD (Singular Value Decomposition). SVD also takes care of round-off errors and is the recommended method according to Press et al. (1992).

Some other quite powerful (gradient and direct search) methods of solving unconstrained nonlinear regression (or more generally nonlinear optimization) problems are: Levenberg-Marquardt, steepest descent/ascent, conjugate gradient search (Fletcher-Reeves), "full Newton-type", etc. (Chapra \& Canale, 2006; Press et al., 1992). In many numerical methods the analytical or approximate knowledge of the Hessian (matrix of second-order partial derivatives) may be helpful. The constrained nonlinear optimization methodology uses many of these aforementioned methods specifically adjusted to meet constraints in a direct or indirect approach. In the case where only one unknown coefficient exists, as is our case here, a resulting single nonlinear equation yields:

$$
\sum_{i=1}^{N}\left[y_{i}-y\left(a_{0} ; x_{i}\right)\right] \frac{d y\left(a_{0} ; x_{i}\right)}{d a_{0}}=0
$$

Since only one positive real root exists (unknown temperature), the Equation A9 can be solved by any of the many available nonlinear equations roots-seeking methods, such as, bisection, secant, Regula-falsi, Newton-Raphson, fixed-point iteration, etc. (Chapra \& Canale, 2006; Press et al., 1992). Replacing into Equation A9, the nonlinear regression ISOAM model is evaluated from:

$$
\begin{aligned}
& \sum_{i=1}^{N}\left[\rho_{I S A}\left(H_{i}\right)-\rho_{\text {ISOAM }}\left(T_{0} ; H_{i}\right)\right] \frac{d \rho_{\text {ISOAM }}\left(T_{0} ; H_{i}\right)}{d T_{0}}=0 \\
& \rho_{\text {ISOAM }}\left(T_{0} ; H\right)=\left(\frac{p_{0}}{R}\right) \cdot \frac{1}{T_{0}} \cdot \exp \left[-\frac{1}{T_{0}} \cdot\left(\frac{g_{0}}{R}\right) \cdot H\right]
\end{aligned}
$$

Known ISA density values are used as observational exact points (with no uncertainty) for which the minimum residual sum of square differences is sought. 


\section{Appendix $B$}

Constrained optimization using Lagrange multipliers

In order to find the parameters of the parabolic temperature profile (NLPAM) with given two constrains at SL and $47 \mathrm{~km}$, a method of Lagrange multipliers or the Lagrangian method of undetermined coefficients (Ashley, 1992; Greenwood, 1997; Lanzos, 1986; Miller, 2000; Pierre, 1986; Tribus, 1961; Widder, 1989) with the least-square-sum performance measure is used. Least-square-sum is essentially a maximum likelihood estimator which yields (Press et al., 1992):

$$
S=\chi^{2}=\sum_{i=1}^{N}\left\{\frac{1}{\sigma_{i}}\left[y_{i}-\sum_{k=1}^{K} a_{k} X_{k}\left(x_{i}\right)\right]\right\}^{2}
$$

Here, $\mathrm{N}$ is the number of equidistant discrete measurements (as a function of discrete independent variable) and $\mathrm{K}$ is the polynomial degree. The standard deviation $\sigma_{i}=1$ (Press et al., 1992) because it is or unknown or the data are exact (based on ISA model). The regression is linear because it describes linear relationship between unknown coefficients $a_{k}$ for the basis-functions $X_{k}$ which in the case of linear polynomial regression are monomials, $X_{k}=x^{k}$. In fact, basis functions can be any other functions, such as, trigonometric functions (e.g., Fourier series), etc. For example, two constraints can be given maximum for a quadratic polynomial:

$$
g_{1}\left(a_{k} ; x_{1}\right)=0 \quad g_{2}\left(a_{k} ; x_{2}\right)=0 \quad(j=1,2, \ldots, J) \quad J<K+1
$$

If too many constrains are given the problem becomes over-constrained and no solution exists. What it means that three points define an interpolating quadratic polynomial regardless of the performance measure. Four constraints would be generally asking too much from a quadratic polynomial and may not be consistent.

A Lagrangian (Ashley, 1992; Greenwood, 1997; Lanzos, 1986) or the augmented performance (Lagrangian) measure (Miller, 2000; Minoux, 1986, Nocedal \& Wright, 2006, Pierre, 1986; Widder, 1989) is now constructed:

$$
L\left(a_{k}, \lambda_{j} ; x\right)=S\left(a_{k} ; x\right)+\sum_{j=1}^{J} \lambda_{j} g_{j}\left(a_{k} ; x\right) \quad J<K+1
$$


In order to find stationary points (maximum, minimum, or inflexion point), the following sufficient and necessary conditions must be satisfied (Greenwood, 1997; Lanzos, 1986; Minoux, 1986, Nocedal \& Wright, 2006; Pierre, 1986; Tribus, 1961; Widder, 1989):

$$
\frac{\partial L}{\partial a_{k}}=0 \quad \frac{\partial L}{\partial \lambda_{j}}=g_{j}=0 \quad(k=0,1,2, \ldots, K \quad j=1,2, \ldots, J)
$$

Often a negative sign is used instead of positive with Lagrange multipliers in Equation $\mathrm{B} 3$ resulting in condition $\nabla S=\lambda \nabla g$. The extremum condition given by Equation $\mathrm{B} 4$ results in $\mathrm{K}+1+\mathrm{J}$ simultaneous linear algebraic equations $(\mathrm{K}+1$ for unknown coefficients and $\mathrm{J}$ for Lagrange multipliers) with the same number of unknowns which can be solved with any of the many existing methods (Carnahan et al., 1969; Chapra \& Canale, 2006; Conte \& de Boor, 1986; Demidovich \& Maron, 1987; Press et al., 1992; Ralston \& Rabinowitz 1978). Generally, one can write:

$$
[B] \cdot\{Z\}=\{C\} \quad \Rightarrow \quad\{Z\}=[B]^{-1} \cdot\{C\}
$$

Matrix B is a non-singular square matrix of known coefficients for which the inverse exists (determinant not zero). The solution vector contains three unknown coefficients, $a_{k}$, which in fact minimize the sum of squares (Equation B1) under given constraints and two Lagrangian multipliers $\lambda_{k}$.

General optimization theories (constrained and unconstrained) are well covered in some references used here (Miller, 2000; Minoux, 1986, Nocedal \& Wright, 2006, Pierre, 1986). Torenbeek (2013) demonstrates applications of various optimization methods in advanced aircraft design. 


\section{Appendix C}

Gauss-Legendre numerical integration method

A definite integral with arbitrary real limits can be converted into a normalized Gauss-Legendre (G-L) quadrature form:

$$
I=\int_{a}^{b} f(x) d x=\left(\frac{b-a}{2}\right) \cdot \int_{-1}^{+1} f\left(\frac{a+b}{2}+\frac{b-a}{2} t\right) d t=\int_{-1}^{+1} g(t) d t=\sum_{i=1}^{n} A_{i} \cdot g\left(t_{i}\right)
$$

where,

$$
x_{i}=\frac{a+b}{2}+\frac{b-a}{2} t_{i} \quad(i=1,2, \ldots, n) \quad g\left(t_{i}\right)=\left(\frac{b-a}{2}\right) \cdot f\left(x_{i}\right)
$$

Unlike in Newton-Cotes integration where the function evaluations are always at the interval boundaries, the Gauss quadrature introduces additional degree-of-freedom by choosing the abscissa points for function evaluations. As a result, the Gauss quadrature is significantly more accurate and requires less evaluations than closed Newton-Cotes formulas (e.g., popular Simpson's 1/3-rd rule). Various orthogonal polynomials are typically used for Gauss quadrature (e.g., Gauss-Legendre, Gauss-Hermite, Gauss-Chebyshev, Gauss-Laguerre). Gauss-Legendre quadrature is particularly popular and powerful. Orthogonal Legendre polynomials can be conveniently defined by the Rodrigues' formula (Lebedev, 1972) in an interval $[-1,+1]$ :

$$
P_{n}(t)=\frac{1}{2^{n} n !} \frac{d^{n}}{d t^{n}}\left[\left(t^{2}-1\right)^{n}\right]
$$

An important orthogonal property that also defines $\mathrm{L}^{2}$ norm of Legendre polynomials:

$$
\int_{-1}^{+1} P_{n}(x) \cdot P_{m}(x) d x=\frac{2}{2 n+1} \delta_{n m} \quad \int_{-1}^{+1} P_{n}^{2}(x) d x=\frac{2}{2 n+1}
$$

Coefficients $A_{i}$ 's are the weights and $t_{i}$ 's are the roots (zeros) of orthogonal Legendre polynomials with some selected useful properties (Demidovich \& Maron, 1987): 
$\sum_{i=1}^{n} A_{i}=2 \quad \sum_{i=1}^{n} A_{i} \cdot t_{i}=0 \quad P_{n}\left(t_{i}\right)=0$

The six-point G-L quadrature formula has six weights and roots of orthogonal Legendre polynomials which are summarized in Table $\mathrm{C} 1$. As a matter of fact, eight-point G-L quadrature was used in our computational programs for increased accuracy. More details on Gauss quadrature and tables of values of zero's and weight coefficients are given in (Carnahan et al., 1969; Chapra \& Canale, 2006; Demidovich \& Maron, 1987; Press et al., 1992).

Using a linear substitution, the mass-fraction integral is first converted into normalized domain of Legendre polynomials and then weighted sum is formed. An eight-point G-L quadrature method involving four positive and four negative zeros of Legendre polynomials with eight weights (four pairs) was actually employed in Equation C1. Integrand was evaluated eight times at specific Legendre polynomial zero points in an interval $[-1,+1]$. Ten-point and fifteenpoint G-L zeros and weight coefficients with 15-significant-digits precision were also used and are given in Carnahan et al. (1969).

Table C1

Weight Coefficients and Zeros of Legendre Polynomials Used in Six-Point GaussLegendre Quadrature

\begin{tabular}{ccccccc}
\hline$i$ & 1 & 2 & 3 & 4 & 5 & 6 \\
\hline$A_{i}$ & 0.17132450 & 0.36076158 & 0.46791394 & 0.46791394 & 0.36076158 & 0.17132450 \\
$t_{i}$ & -0.9326951 & -0.66120939 & -0.2386192 & +0.2386192 & +0.66120939 & +0.9326951 \\
\hline
\end{tabular}

Note. Adapted from Demidovich and Maron (1987)

To calculate the scale heights of NLPAM for specific mass fractions $f$ (50\%, $75 \%, 90 \%$, etc., atmosphere), a complicated iterative solution of the inverse problem is sought. A positive real roots of nonlinear algebraic-integral equation is found by halving the interval, localizing the solution, and minimizing the residual function $g(H)$ :

$$
f \cdot M_{0}-M(H)=\xi(H) \quad f \in[0,1) \quad \xi(H) \rightarrow 0 \quad H \rightarrow H_{f}
$$




\section{References}

Ambaum, M. H. P. (2008). General relationships between pressure, weight and mass of a hydrostatic fluid. Proceedings of the Royal Society A, 464, 943950. doi: 10.1098/rspa.2007.0148

Ashley, H. (1992). Engineering analysis of flight vehicles. Mineola, NY: Dover.

Azad, R. S., \& Kassab, S. Z. (1989). New method of obtaining dissipation. Experiments in Fluids, 7, 81-87. doi: 10.1007/BF00207299

Boltzmann, L. (1964). Lectures on gas theory (translated from original in German “Vorlesungenen über Gastheorie”, 1896/1898, Leipzig). Berkley, CA: University of Califormia Press.

Carnahan, B., Luther, H. A., \& Wilkes, J. O. (1969). Applied numerical methods. New York, NY: John Wiley \& Sons.

Chadwick, P. (1999). Continuum mechanics: concise theory and problems. Mineola, NY: Dover.

Chapman, D. R. (1958). An approximate analytical method for studying entry into planetary atmospheres (NACA TN-4276). Ames, CA: National Aeronautics and Space Administration.

Chapra, S. C., \& Canale, R. P. (2006). Numerical methods for engineers (5th ed.). Boston, MA: McGraw-Hill.

Conte, S. C., \& de Boor C. (1986). Elementary numerical analysis: An algorithmic approach, (3rd int. ed.). Singapore, Singapore: McGraw-Hill.

Daidzic, N. E. (1992a, March-April). Droplet breakup in turbulent flow fields. Paper presented at the Sixth Workshop on Two-Phase Flow Predictions. Erlangen, Germany. doi: 10.1002/ppsc.19910080144

Daidzic, N. E., Melling, A., Domnick, J., \& Durst F. (1992b, September-October). Droplet stability in isotropic turbulence. Paper presented at ILASSEurope-92, Eighth Annual European Conference, Institute for Liquid Atomization and Spray Systems, Amsterdam, Netherlands. 
Daidzic, N. E., \& Simones, M. (2010). Aircraft decompression with installed cockpit security door, Journal of Aircraft, 47(2), 490-504. doi: $10.2514 / 1.41953$

Daidzic, N. E. (2015). Efficient general computational method for estimation of standard atmosphere parameters. International Journal of Aviation Aeronautics, and Aerospace, 2(1), 1-35.

Davies, M. (Ed.) (2003). The standard handbook for aeronautical and astronomical engineers. New York, NY: McGraw-Hill.

Demidovich, B. P., \& Maron, I.A. (1987). Computational mathematics. Moscow, Russia: MIR.

Dwight, H. B. (1961). Tables of integrals and other mathematical data (4th ed.). New York, NY: Macmillan.

Greenwood, D. T. (1997). Classical dynamics. Mineola, NY: Dover.

Hansen, C. F. (1976). Molecular physics of equilibrium gases (NASA SP-3096). Washington, DC: National Aeronautics and Space Administration.

Hill, T. L. (1987). Statistical mechanics: principles and selected applications. Mineola, NY: Dover.

Holman, J. P. (1980). Thermodynamics (3rd ed.). Singapore, Singapore: McGrawHill.

Houghton, J. (2002). The physics of atmosphere (3rd ed.). Cambridge, United Kingdom: University Press.

International Civil Aviation Organization (ICAO). (1993). Manual of the ICAO standard atmosphere (extended to 80 kilometers (262 500 feet)) (Doc 7488-CD, 3rd ed.). Montreal, Canada: Author.

International Organization for Standardization (ISO). (1975). Standard atmosphere (ISO 2533:1975/Add. 2: 1997(En)). Geneva, Switzerland: Author. 
Iribarne, J. V., \& Cho, H.-R. (1980). Atmospheric physics. Dordrecht, Holland: D. Reidel.

Kennard, E. H. (1938). Kinetic theory of gases. New York, NY: McGraw-Hill.

Lanczos, C. (1986). The variational principles of mechanics (4th ed.). Mineola, NY: Dover.

Landau, L. D., \& Lifshitz, E. M. (1987). Fluid Mechanics (6th ed.). Oxford, UK: Butterworth Heinemann.

Lebedev, N. N. (1972). Special functions and their applications. Mineola, NY: Dover.

Miller, R. E. (2000). Optimization: Foundations and applications. New York, NY: John Wiley \& Sons.

Minoux, M. (1986). Mathematical programing; Theory and algorithms. Chichester, West Sussex, United Kingdom: John Wiley \& Sons.

National Oceanic and Atmospheric Administration (NOAA). (1976). U.S. standard atmosphere, 1976 (NOAA-S/T 76-1562). Washington, DC: U.S. Government Printing Office.

Nocedal, J., \& Wright, S. J. (2006). Numerical optimization (2nd ed.). New York, NY: Springer.

Pai, Shih-I. (1981). Modern fluid mechanics. New York, NY: Van Nostrand Reinhold.

Pierre, D. A. (1986). Optimization theory with applications. Mineola, NY: Dover.

Press, W. H, Teulkolsky, S. A., Vetterling, W. T., \& Flannery, B. P. (1992). Numerical recipes in FORTRAN: The art of scientific computing (2nd ed.). Cambridge, UK: University Press.

Ralston, A., \& Rabinowitz, P. (1978). A first course in numerical analysis (2nd ed.). New York, NY: McGraw-Hill. 
Reif, F. (1965). Fundamentals of statistical and thermal physics. New York, NY: McGraw-Hill.

Saad, M. (1966). Thermodynamics for engineers. Englewood Cliffs, NJ: Prentice Hall.

Sears, F. W. (1964). An introduction to thermodynamics, the kinetic theory of gases, and statistical mechanics (2nd ed.). Reading, MA: AddisonWesley.

Seber, G. A., \& Wild, C. J. (1989). Nonlinear regression. New York, NY: John Wiley \& Sons.

Spiegel, M. R., \& Liu, J. (1999). Mathematical handbook of formulas and tables (2nd ed.). New York, NY: McGraw-Hill.

Stacey, F. D., \& Davis, P. M. (2008). Physics of the earth (4th ed.). Cambridge, United Kingdom: University Press.

Tennekes, H., \& Lumley, J. L. (1980). A first course in turbulence. Cambridge, MA: The MIT Press.

Tewari, A. (2007). Atmospheric and space flight dynamics: Modelling and simulation with MATLAB® and Simulink®. New York, NY: Birkhäuser.

Torenbeek, E. (2013). Advanced aircraft design: Conceptual design, technology and optimization of subsonic civil airplanes. Chichester, West Sussex, United Kingdom: John Wiley \& Sons.

Trenberth, K. E. (1981). Seasonal variations in global sea level pressure and the total mass of the atmosphere. Journal of Geophysical Research, 86(C6), 5238-5246. doi: 10.1029/JC086iC06p05238

Trenberth, K. E., \& Guillemot, C. J. (1994). The total mass of the atmosphere. Journal of Geophysical Research, 99(D11), 23079-23088. doi: 10.1029/94JD02043

Trenberth, K. E., \& Smith, L. (2005). The mass of the atmosphere: A constraint on global analysis. Journal of Climate, 18, 864-875. doi: 10.1175/JCLI3299.1 
Tribus, M. (1961). Thermostatics and thermodynamics. Princeton, NJ: D. Van Nostrand.

Wallace, J. M., \& Hobbs, P. V. (2006). Atmospheric science: An introductory survey (2nd ed.). Burlington, MA: Academic Press.

Wannier, G. H. (1987). Statistical physics. Mineola, NY: Dover.

Widder, D. V. (1989). Advanced calculus (2nd ed.). Mineola, NY: Dover. 